\title{
HLA-DQ $\beta 1$ alleles associated with Epstein-Barr virus (EBV) infectivity and EBV gp42 binding to cells
}

Qingxue Li, ${ }^{1}$ Wei Bu, ${ }^{1}$ Erin Gabriel, ${ }^{2}$ Fiona Aguilar, ${ }^{1}$ Yo Hoshino, ${ }^{1}$ Hiroko Miyadera, ${ }^{3,4}$ Christoph Hess, ${ }^{5}$ Ronald L. Hornung, ${ }^{6}$ Amitava Roy, ${ }^{7}$ and Jeffrey I. Cohen ${ }^{1}$

${ }^{1}$ Medical Virology Section, Laboratory of Infectious Diseases, ㄹivision of Clinical Research, Biostatistics Research Branch, National Institute of Allergy and Infectious Diseases, NIH, Bethesda, Maryland, USA. ${ }^{3}$ Department of Human Genetics, Graduate School of Medicine, University of Tokyo, Tokyo, Japan. ${ }^{4}$ Research Center for Hepatitis and Immunology, National Center for Global Health and Medicine, Chiba, Japan. ${ }^{5}$ mmunobiology Laboratory, Department of Biomedicine, and Medical Outpatient Division, University Hospital Basel, Basel, Switzerland. 'Clinical Services Program, Leidos Biomedical Research, Inc., Frederick National Laboratory for Cancer Research, Frederick, Maryland, USA. ${ }^{7 B i o i n f o r m a t i c s ~ a n d ~ C o m p u t a t i o n a l ~}$ Biosciences Branch, Rocky Mountain Laboratories, NIH, Hamilton, Montana, USA.

Epstein-Barr virus (EBV) infects B cells and 95\% of adults are infected. EBV glycoprotein gp42 is essential for entry of virus into $B$ cells. EBV gp42 binds to the $\beta 1$ chain of HLA-DQ, -DR, and -DP on $B$ cells, and uses these molecules for infection. To investigate if certain HLA-DQ alleles are associated with EBV seronegativity, we recruited 3,300 healthy adult blood donors, identified 106 EBV-seronegative individuals, and randomly selected a control group of EBV-seropositive donors from the donor pool. A larger than expected proportion of EBV-seronegative subjects were HLA-DQ $\beta 1{ }^{*} 04 /{ }^{*} 05$ and ${ }^{*} 06 /{ }^{*} 06$, and to a lesser extent, ${ }^{*} 02 /{ }^{*} 03$, compared with the control group, while a larger than expected portion of EBV-seropositive persons were HLA-DQ $\beta 1{ }^{*} 02 /{ }^{*} 02$. We examined the ability of EBV gp42 to bind to different HLA-DQ molecules using human and mouse cells stably expressing these alleles. EBV gp42 bound less effectively to cells expressing HLA-DQ $\beta 1{ }^{*} 04 /{ }^{*} 05$, ${ }^{*} 06 /{ }^{*} 06$, or ${ }^{*} 03 /{ }^{*} 03$ than to cells expressing HLA-DQ $\beta 1{ }^{*} 02 /{ }^{*} 02$. These data are consistent with our observations of increased EBV seronegativity with $D Q \beta 1{ }^{*} 04 /{ }^{*} 05$ or ${ }^{*} 06 /{ }^{*} 06$ alleles. These findings emphasize the importance of a single genetic locus (HLA-DQ $\beta 1$ ) to influence infectivity with EBV.

Conflict of interest: The authors have declared that no conflict of interest exists.

Submitted: November 25, 2015 Accepted: January 5, 2017 Published: February 23, 2017

Reference information: JCI Insight. 2017;2(4):e85687. https:// doi.org/10.1172/jici.insight.85687.

\section{Introduction}

Over $95 \%$ of adults are infected with Epstein-Barr virus (EBV) worldwide. Although the infection often presents with nonspecific or no symptoms in young children, EBV frequently causes infectious mononucleosis in young adults (1). EBV is associated with a number of malignancies, including Burkitt and Hodgkin lymphoma, nasopharyngeal carcinoma, and gastric carcinoma. In immune-deficient patients EBV can result in lymphoproliferative disease.

The reservoir for EBV infection in humans is the B lymphocyte, which is the site of latent infection and virus persistence. The critical role for $\mathrm{B}$ cells in EBV infection is demonstrated by the observation that persons who lack mature B cells cannot be infected with EBV (2). EBV encodes numerous glycoproteins on its envelope that participate in virus entry into cells, including glycoproteins gp350, gB, gH/gL, and gp42 (3). The initial virus attachment onto B cells is mediated through gp350 binding to its cellular ligand CD21 (also known as CR2 or C3d receptor) or CD35 (also known as CR1) (4-6). A complex of 4 glycoproteins, $\mathrm{gH} / \mathrm{gL}, \mathrm{gB}$, and gp42, is required for fusion of the viral envelope with the cell plasma membrane. EBV gp42 utilizes HLA class II molecules as a coreceptor to infect B cells $(7,8)$. EBV gp42, a type II membrane glycoprotein, interacts with $\mathrm{gH} / \mathrm{gL}$ through its $\mathrm{N}$-terminal domain. The $\mathrm{C}$-terminus of gp42 bears a similarity with C-type lectin domains and is important for binding to the $\beta$ chain of $\operatorname{HLA}$ class II $(9,10)$. Blocking the interaction between gp42 and HLA class II with antibodies against either of these proteins, or with soluble gp42 protein, impairs EBV infection of B cells (7).

HLA class II molecules are composed of 2 polypeptide chains ( $\alpha$ and $\beta$ ). Each $\alpha$ and $\beta$ chain has 2 domains - a highly conserved $\alpha 2$ and $\beta 2$ region and a highly polymorphic $\alpha 1$ and $\beta 1$ domain. The antigenpeptide-binding groove is positioned between domains $\alpha 1$ and $\beta 1$ (11). HLA class II molecules are encoded 
Table 1. Population distribution by ethnic group

\begin{tabular}{lccccc}
\hline Ethnic Group & \multicolumn{2}{c}{ EBV Seronegative } & & \multicolumn{2}{c}{ EBV Seropositive } \\
European descent & $n$ & $\%$ & $n$ & $\%$ \\
African American & 84 & 79.2 & 121 & 55.5 \\
Other & 15 & 14.2 & 79 & 36.2 \\
Total & 7 & 6.6 & 18 & 8.3 \\
\hline
\end{tabular}

by 3 different loci, HLA-DR, -DQ, and -DP, which share approximately $70 \%$ amino acid identity with each other and are inherited as haplotypes. Previous studies have shown that all 3 HLA class II molecules, HLADR, HLA-DQ, and HLA-DP, can serve as receptors for EBV gp42 $(9,12)$. While peptide antigen binding to the peptide pocket of HLA class II involves both the $\alpha 1$ and $\beta 1$ subunits of the $\alpha \beta$ heterodimer, gp42 interacts only with the $\beta 1$ subunit of $\operatorname{HLA}$ class II $(9,10,13)$. Nevertheless, soluble gp42 inhibits antigen presentation $(9,14,15)$, possibly by blocking the interaction between the T cell receptor and the HLA-peptide antigen complex (15).

About $5 \%$ of adults are seronegative for EBV throughout their lifetime. It is generally assumed that selection for resistance to infection drives evolution of MHC variation (16). This seems paradoxical for EBV, which has evolved to utilize HLA class II to facilitate entry and infection. Therefore, determining which HLA-DQ alleles are associated with EBV infectivity or resistance to EBV infection is important to better understand how the virus has evolved with MHC molecules. A previous study using transiently expressed HLA-DQ in a human lymphoblastoid cell line (LCL) lacking HLA class II found that cells expressing HLA-DQ2 $\left(\alpha^{*} 0501 \times \beta^{*} 0201\right)$ were more susceptible to infection with a genetically modified laboratory strain of EBV, while HLA-DQ3.3-expressing $\left(\alpha^{*} 0301 \times \beta^{*} 03032\right)$ cells were resistant to infection, and suggested a coreceptor restriction within the HLA-DQ locus for EBV infection (17). However, since the LCL used has a large homozygous deletion in the HLA class II and HLA-DM coding regions, and is deficient in the assembly and transport of class I molecules to the cell surface, it is not clear if the observed coreceptor restriction applies to cells without such mutations and to humans that are infected with wild-type viruses. To address these questions, we identified $106 \mathrm{EBV}$-seronegative individuals from a pool of about 3,300 healthy blood donors and performed genotyping for the HLA-DQ $\beta$ chain. Our results indicate that HLA-DQ alleles influence EBV infectivity in naturally infected humans. Subsequent in vitro binding and infectivity assays showed that the specificity of EBV gp42 for binding to specific HLA-DQ alleles was sufficient to account for allele-specific correlations with EBV seropositivity and seronegativity in naturally infected humans.

\section{Results}

The HLA-DQ $\beta 1{ }^{*} 02 / * 02$ allele correlates with EBV seropositivity, while HLA-DQ $\beta 1{ }^{*} 04 /{ }^{*} 05, H L A-D Q \beta 1$ ${ }^{*} 06 /{ }^{*} 06$, and $D Q \beta 1 * 02 / * 03$ correlate with EBV seronegativity. About 3,300 healthy adult blood donors were recruited from the United States and Switzerland and 106 were identified as seronegative for EBV. In addition, 218 blood donors who had been part of the screening process to identify seronegative donors in both countries, but were found to be EBV seropositive were randomly selected to serve as controls. Table 1 shows the ethnicity of the EBV-seronegative donors and the seropositive controls that were blindly selected from the EBV-seropositive candidate pool. Consistent with prior reports (18-21), we observed that the EBV-seronegative group had a higher percentage of subjects of European descent than African Americans and other non-white individuals, compared with the EBV-seropositive group.

We determined HLA-DQ alleles for each of the 324 blood donors (Table 2). Three HLA-DQ $\beta 1$ alleles were associated with increased odds of EBV seronegativity, and 1 with decreased odds of EBV seronegativity. All 4 subjects with DQ $\beta 1 * 04 /{ }^{*} 05$ were EBV seronegative (unadjusted Fisher exact $P$ value $=0.011$, Table 3). There was a higher than expected proportion of subjects with DQ $\beta 1{ }^{*} 06 /{ }^{*} 06$ and DQ $\beta 1 * 02 /{ }^{*} 03$ among the EBV-seronegative persons compared with the seropositive group (for DQ $\beta 1 * 06 / * 06$ odds ratio 2.62 , unadjusted Fisher exact $P$ value $=0.03$; for DQ $\beta 1{ }^{*} 02 /{ }^{*} 03$ odds ratio $1.79, P$ value $=0.087$ ). All 12 persons with the DQ $\beta 1{ }^{*} 02 /{ }^{*} 02$ genotype were EBV seropositive (unadjusted Fisher exact $P$ value $=$ 
Table 2. Frequency of the 15 combinations of the 2 HLA-DQ $\beta 1$ alleles among EBV seronegative blood donors (top panel) and seropositive controls (bottom panel)

\begin{tabular}{|c|c|c|c|c|c|c|c|c|}
\hline \multicolumn{2}{|c|}{ EBV Seronegative } & \multicolumn{7}{|c|}{$\beta 1$ - 2nd Alleles } \\
\hline \multirow{6}{*}{$\beta 1-1$ st Alleles } & & ${ }^{*} 02$ & ${ }^{*} 03$ & ${ }^{*} 04$ & ${ }^{*} 05$ & ${ }^{*} 06$ & $n$ & $\%$ \\
\hline & ${ }^{*} 02$ & 0 & 20 & 2 & 10 & 4 & 36 & 34.0 \\
\hline & ${ }^{*} 03$ & - & 14 & 0 & 6 & 16 & 36 & 34.0 \\
\hline & ${ }^{*} 04$ & - & - & 0 & 4 & 1 & 5 & 4.7 \\
\hline & ${ }^{*} 05$ & - & - & - & 3 & 13 & 16 & 15.1 \\
\hline & *06 & - & - & - & - & 13 & 13 & 12.3 \\
\hline
\end{tabular}

\begin{tabular}{|c|c|c|c|c|c|c|c|c|}
\hline \multicolumn{2}{|c|}{ EBV Seropositive } & \multicolumn{7}{|c|}{$\beta 1$ - 2nd Alleles } \\
\hline \multirow{5}{*}{$\beta 1-1$ st Alleles } & & ${ }^{*} 02$ & ${ }^{*} 03$ & ${ }^{*} 04$ & *05 & ${ }^{*} 06$ & $n$ & $\%$ \\
\hline & ${ }^{*} 02$ & 12 & 25 & 7 & 22 & 20 & 86 & 39.4 \\
\hline & ${ }^{*} 03$ & - & 25 & 4 & 18 & 33 & 80 & 36.7 \\
\hline & ${ }^{*} 05$ & - & - & - & 5 & 27 & 32 & 14.7 \\
\hline & ${ }^{*} 06$ & - & - & - & - & 11 & 11 & 5.0 \\
\hline
\end{tabular}

0.011). However, when the $P$ values were adjusted for multiple comparisons considering all 15 DQ genotypes using false discovery rate adjustment they were no longer significant. This reflects the need for a larger sample size, since HLA-DQ is highly polymorphic and allele frequencies in the general population vary.

When we analyzed the data for an association between EBV serostatus and the expression of any single HLA-DQ $\beta 1$ allele, we found that persons with at least one HLA-DQ $\beta 1 * 03$ allele had the highest observed odds of being seronegative (odds ratio 1.21). We also observed that there was a higher proportion of EBVseropositive individuals who carried at least one DQ $\beta 1 * 02$ or one DQ $\beta 1{ }^{*} 04$ allele (odds ratio 0.79 and 0.70 , respectively); however, these associations did not reach statistical significance (Table 4 ).

EBV gp42 protein binds more efficiently to $H L A-D Q \beta 1 * 02 /{ }^{*} 02-$ positive cells than to $H L A-D Q \beta 1 * 03 /{ }^{*} 03$ positive or ${ }^{*} 04 /{ }^{*} 05$-positive cells. The major target of EBV infection in vivo is B cells, and EBV utilizes HLA

Table 3. Statistical analysis of HLA-DQ $\beta 1$ genotype among EBV-seronegative and -seropositive blood donors

\begin{tabular}{|c|c|c|c|c|c|c|c|}
\hline \multirow[t]{2}{*}{$n$} & \multicolumn{2}{|c|}{ EBV-Seronegative Donors } & \multicolumn{2}{|c|}{ EBV-Seropositive Controls } & \multicolumn{3}{|c|}{ Statistical Analysis } \\
\hline & $n$ & $\%$ & $n$ & $\%$ & $\begin{array}{l}\text { Odds Ratio } \\
\text { Seronegative }\end{array}$ & Fisher Exact $P$ value & Adjusted $P$ for FDR \\
\hline 45 & 20 & 18.87 & 25 & 11.47 & 1.792 & 0.087 & 0.325 \\
\hline 9 & 2 & 1.89 & 7 & 3.21 & 0.581 & 0.723 & 0.986 \\
\hline 32 & 10 & 9.43 & 22 & 10.09 & 0.928 & 1 & 1 \\
\hline 39 & 14 & 13.21 & 25 & 11.47 & 1.174 & 0.716 & 0.986 \\
\hline 4 & 0 & 0.00 & 4 & 1.83 & 0 & 0.307 & 0.659 \\
\hline 24 & 6 & 5.66 & 18 & 8.26 & 0.667 & 0.501 & 0.94 \\
\hline 49 & 16 & 15.09 & 33 & 15.14 & 0.997 & 1 & 1 \\
\hline 2 & 0 & 0.00 & 2 & 0.92 & 0 & 1 & 1 \\
\hline 4 & 4 & 3.77 & 0 & 0.00 & $\infty$ & 0.011 & 0.083 \\
\hline 24 & 13 & 12.26 & 11 & 5.05 & 2.622 & 0.025 & 0.123 \\
\hline 324 & 106 & 100.00 & 218 & 100.00 & & & \\
\hline
\end{tabular}

FDR, false discovery rate. Bold indicates statistically significant for Fischer exact $P$ value. 
Table 4. Statistical analysis of HLA-DQ $\beta 1$ allele frequency among EBV-seronegative and -seropositive blood donors

\begin{tabular}{|c|c|c|c|c|c|}
\hline \multicolumn{6}{|c|}{ Donors with at least 1 allele } \\
\hline & \multicolumn{2}{|c|}{ EBV Seronegative } & \multicolumn{2}{|c|}{ EBV Seropositive } & \multirow{2}{*}{$\begin{array}{c}\text { Odds Ratio } \\
\text { (Seronegative) }\end{array}$} \\
\hline & $n$ & $\%$ of Seronegative & $n$ & $\%$ of Seropositive & \\
\hline $\mathrm{DQ} \beta 1^{*} 02$ & 36 & 34 & 86 & 39.5 & 0.790 \\
\hline $\mathrm{DQ} \beta 1^{*} 03$ & 56 & 52.8 & 105 & 48.2 & 1.205 \\
\hline$D Q \beta 1{ }^{*} 04$ & 7 & 6.6 & 20 & 9.2 & 0.701 \\
\hline $\mathrm{DQ} \beta 1^{*} 05$ & 36 & 34 & 72 & 33 & 1.043 \\
\hline $\mathrm{DQ} \beta 1^{*} 06$ & 47 & 44.3 & 98 & 45 & 0.976 \\
\hline
\end{tabular}

class II as a coreceptor for virus entry into B cells mediated by EBV gp42 binding to HLA class II molecules. To further investigate the contribution of HLA-DQ to EBV infectivity, we studied the ability of EBV gp42 to bind to different HLA-DQ alleles. LCLs with HLA-DQ $\beta 1{ }^{*} 02 /{ }^{*} 02$ (IHW09086), DQ $\beta 1$ *03/*03 (IHW09035), and DQ $\beta 1 * 04 / * 05$ (IHW01021 and IHW01019) (Table 5) were stained with antiHLA-DQ antibody Ia3 and each expressed similar levels of the DQ protein on the cell surface (Figure 1). Jurkat cells (a T cell leukemia cell line), which do not express HLA-DQ on their surface (22), served as a negative control. Purified gp42-His was incubated on ice with the cells and binding was quantified using anti-His antibody and FACS. Cells expressing HLA-DQ $\beta 1 * 02 /{ }^{*} 02$ bound gp 42 more efficiently than cells expressing DQ $\beta 1 * 03 /{ }^{*} 03$ or DQ $\beta 1 * 04 /{ }^{*} 05$ (unadjusted Wilcoxon signed-rank $P$ value $=0.03$ for DQ $\beta 1$ ${ }^{*} 02 /{ }^{*} 02$ vs. ${ }^{*} 03 /{ }^{*} 03 ; P=0.002$ for DQ $\beta 1{ }^{*} 02 /{ }^{*} 02$ vs. ${ }^{*} 04 /{ }^{*} 05$; Figure 2 ). In contrast, no significant difference in gp42 binding between DQ $\beta 1{ }^{*} 03 /{ }^{*} 03$ and DQ $\beta 1{ }^{*} 04 /{ }^{*} 05$ was observed (Wilcoxon rank-sum test $P$ value $=0.118$ for either of the DQ $\beta 1{ }^{*} 04 /{ }^{*} 05$ cell lines). While both DQ $\beta 1{ }^{*} 04 /{ }^{*} 05$ cell lines bound less gp42 than the DQ $\beta 1{ }^{*} 02 /{ }^{*} 02$ cell line, there was a consistent difference in binding between the two DQ $\beta 1$ $* 04 /{ }^{*} 05$ cell lines over multiple experiments, although the difference did not reach statistical significance (unadjusted Wilcoxon signed-rank $P$ value $=0.06$ ). Since the 2 cell lines have the identical genotypes for HLA-DQ $\alpha 1 / \beta 1$, -DP $\alpha 1 / \beta 1$, and -DR $\beta 1$, this indicates that other genetic factors, such as HLA-DR $\beta 3, \beta 4$, and $\beta 5$, likely affect binding of gp42.

We also tested gp42 binding using purified gp42-Fc, in which IgG1 Fc was fused to the N-terminus of gp42 (a type II membrane protein) (9). A previous study showed that gp42-Fc blocks EBV infection at the entry step (7). Unlike gp42-His, which is a monomer, gp42-Fc forms a dimer through its Fc domain. To minimize nonspecific binding mediated through cell surface $F_{c}$ receptor ( $\left.F c R\right)$, a construct with a mutant Fc coding sequence was used that has markedly diminished ( $>10,000$-fold less) FcR binding activity (9), and an FcR blocking solution was added before incubation with anti-human IgG antibody to reduce background binding. To further control for the Fc domain fused to gp42, purified IgG-Fc protein was used as an internal control, and gp42 binding was calculated by subtraction of the background binding observed with the internal control. Similar to the results observed with gp42-His, binding of gp42-Fc to cells expressing DQ $\beta 1{ }^{*} 02 /{ }^{*} 02$ was significantly increased as compared with cells expressing DQ $\beta 1{ }^{*} 03 /{ }^{*} 03$ or DQ $\beta 1{ }^{*} 04 /{ }^{*} 05$ (unadjusted Wilcoxon signed-rank $P$ value $=0.008$ for DQ $\beta 1{ }^{*} 02 /{ }^{*} 02$ vs. ${ }^{*} 03 /{ }^{*} 03 ; P=0.002$ for DQ $\beta 1{ }^{*} 02 /{ }^{*} 02$ vs. ${ }^{*} 04 /{ }^{*} 05$; Figure 3 ). In contrast, no significant difference was observed for gp $42-\mathrm{Fc}$ binding between DQ $\beta 1{ }^{*} 03 /{ }^{*} 03$ and ${ }^{*} 04 /{ }^{*} 05$ (unadjusted Wilcoxon signed-rank $P$ value $=0.2$ for DQ $\beta 1$ ${ }^{*} 03 /{ }^{*} 03$ vs. $\left.{ }^{*} 04 /{ }^{*} 05\right)$. The results of both the gp 42 -His and gp $42-\mathrm{Fc}$ binding experiments, showing that EBV gp42 has increased binding to cells expressing DQ $\beta 1 * 02 /{ }^{*} 02$ compared with cells expressing DQ $\beta 1{ }^{*} 03 /{ }^{*} 03$ or DQ $\beta 1{ }^{*} 04 /{ }^{*} 05$, are consistent with the associations of HLA-DQ $\beta 1$ types with the EBV serostatus of blood donors. These data provide evidence of the importance of EBV gp42 binding to DQ $\beta 1$ as a predictor of EBV seronegativity.

EBV gp 42 protein binds more efficiently to $H L A-D Q \beta 1 * 02 / * 02$-positive and *05/*05-positive cells than to $H A L$ $D Q \beta 1 * 03 /{ }^{*} 03-$ positive and $* 06 /{ }^{*} 06$-positive cells when expressed in the absence of other HLA molecules. In addition to HLA-DQ, EBV gp42 can also bind to DR and DP $(7,12)$. This may explain the variation in binding efficiency observed with cell lines that express identical DQ $\beta 1$ alleles, such as IHW01021 and IHW01019 (Figure 2). In addition, although structural studies suggested that gp42 interacts with HLA $\beta$ chain only (9, 

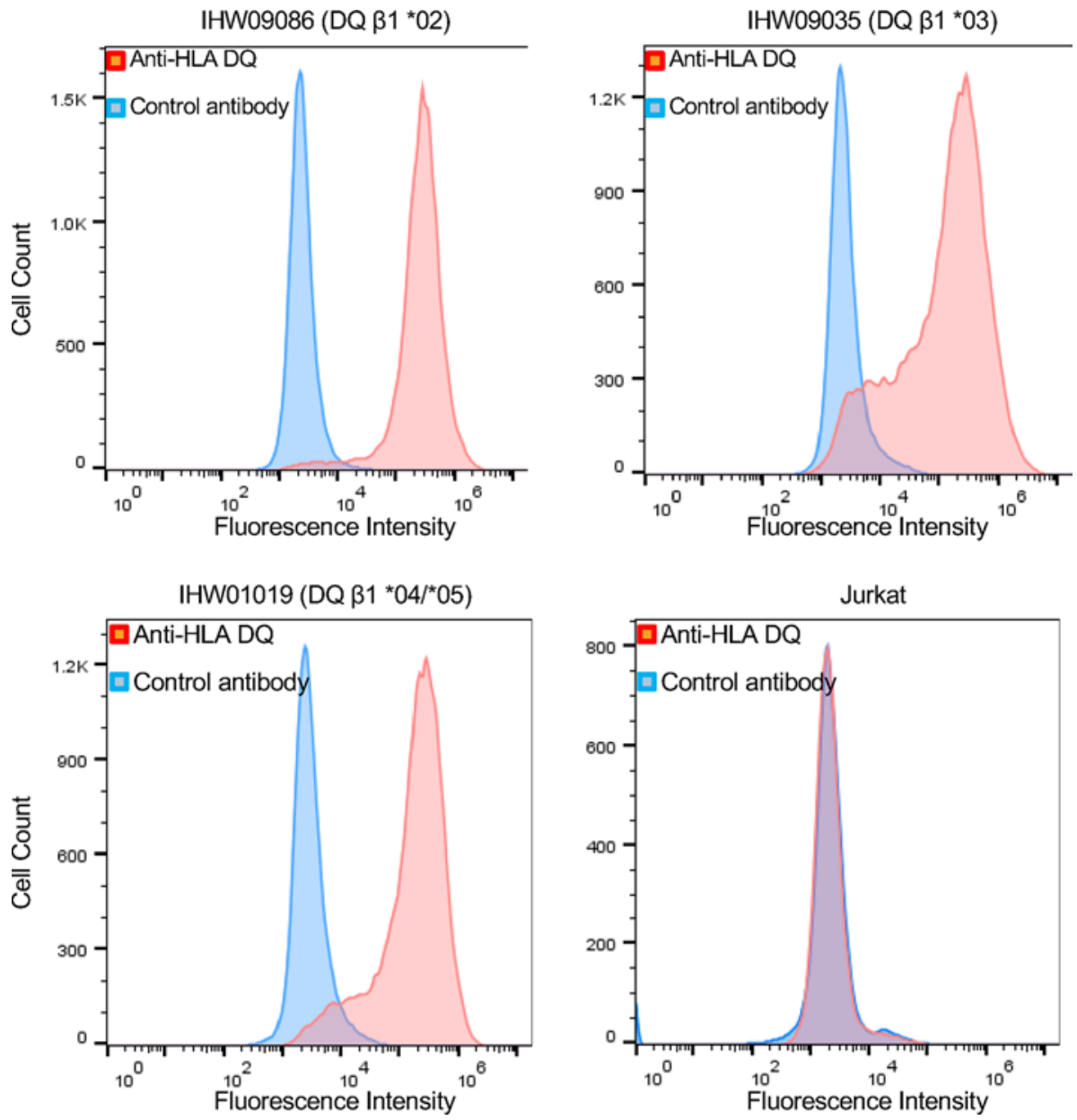

Figure 1. Lymphoblastoid cell lines (LCLs) used in gp42 binding assays express similar levels of HLA-DQ on their cell surface. LCLs or Jurkat $T$ cells were incubated with mouse anti-HLA-DQ antibody la3 on ice followed by an anti-mouse secondary antibody conjugated with Alexa488. After fixation, fluorescence intensity was measured by flow cytometry.

10, 23), HLA $\alpha$ chains may be important, since the proper pairing of $\alpha$ and $\beta$ subunits is important to maintain stability of HLA $\alpha \beta$ heterodimers (24). Therefore, we constructed mouse cell lines stably expressing human HLA-DQ alleles, in the absence of other HLA proteins, and performed gp42 binding assays. Mouse 3T3 cells were transduced with retroviruses encoding various combinations of $\mathrm{DQ} \alpha$ alleles (also expressing GFP) and DQ $\beta$ alleles (also expressing a puromycin-resistance gene); cell lines expressing the DQ proteins were selected by their resistance to puromycin, and by FACS sorting for GFP-positive cells. The resulting cells were greater than 90\% GFP positive. Cell surface DQ expression was measured by staining with antiDQ Ia3. The resulting ratio of cell surface DQ expression to internal control GFP was designated as $\triangle \mathrm{MHC}$, which is a function of intrinsic stability of the DQ pro-

teins, the combined outcome of $\alpha \beta$ heterodimer assembly, transportation to the cell surface, and the rate of DQ protein turnover (24). HLA $\alpha \beta$ heterodimer stability on the cell surface $(\triangle \mathrm{MHC})$ varied among different DQ $\alpha \beta$ heterodimers (Table 6). To provide an interassay control, we normalized $\triangle \mathrm{MHC}$ for each HLA-DQ $\alpha \beta$ heterodimer against that of DQ $\alpha 1^{*} 01: 01 / \beta 1^{*} 06: 02$ (and therefore assigned the normalized $\triangle \mathrm{MHC}$ for DQ $\alpha 1^{*} 01: 01 / \beta 1 * 06: 02$ as 1 ), since DQ $\alpha 1^{*} 01: 02 / \beta 1 * 06: 02$ has been reported to be highly stable, even in the presence of $\operatorname{SDS}(24,25)$. In agreement with prior work, $\alpha 1^{*} 01: 01 / \beta 1 * 06: 02$ was the most stable DQ molecule in our study (Table 6). A previous study of DQ alleles in the general population showed high haplotype and phenotype frequencies for DQ $\alpha 1^{*} 05: 01 / \beta 1 * 02: 01, \alpha 1^{*} 05: 01 / \beta 1 * 03: 01, \alpha 1^{*} 04: 01 / \beta 1 * 04: 02$, $\alpha 1^{*} 01: 01 / \beta 1^{*} 05: 01$, and $\alpha 1^{*} 01: 01 / \beta 1^{*} 06: 02$ (26) (NCBI dbMHC database, https://www.ncbi.nlm.nih. $\mathrm{gov} / \mathrm{gv} / \mathrm{mhc} /$ ). Therefore, we used cells stably expressing these DQ alleles for the gp42-His binding assay. gp42-His protein was incubated with cells and detected with anti-His mouse antibody. Surface DQ expression was quantified, $\triangle \mathrm{MHC}$ was calculated, and gp42 binding efficiency was defined as the ratio of the mean fluorescence intensity of gp42 binding divided by $\triangle \mathrm{MHC}$. HLA-DQ $\beta 1^{*} 02: 01$ bound gp42 with higher efficiency than $\beta 1^{*} 03: 01$ (unadjusted Wilcoxon signed-rank $P$ value $<0.001$, Figure 4 and Table 7). HLA-DQ $\beta 1^{*} 06: 02$ bound gp 42 with low efficiency, similar to that of $\beta 1^{*} 03: 01$ (unadjusted Wilcoxon signed-rank $P$ value $=0.353$ for $\beta 1^{*} 03: 01$ vs. $\beta 1 * 06: 02 ; P=0.001$ for $\beta 1^{*} 02: 01$ vs. $\left.\beta 1^{*} 06: 02\right)$ (Table 7). Taken together, these results are consistent with those obtained for gp42 binding to the human cell lines and our observations for EBV serostatus and HLA-DQ $\beta 1$ types in blood donors.

$H L A-D Q \beta 1 * 02 / * 02$ alleles facilitate EBV infectivity more efficiently than other alleles. In our cohort of healthy donors, all individuals with a DQ $\beta 1{ }^{*} 02 /{ }^{*} 02$ genotype were EBV seropositive (Table 3 ). In addition, this genotype mediated gp42 binding on human cells more efficiently than other alleles (Figures 2 and 3). To determine if the enhanced gp42 binding associated with DQ $\beta 1 * 02 /{ }^{*} 02$ correlates with increased EBV infectivity, we cloned $\mathrm{DQ} \alpha$ and $\beta$ chains into vectors, expressed these genes in human cells, and verified that the mature DQ $\alpha 1 / \beta 1$ heterodimers were expressed on the cell surface ( $\mathrm{Li}$ et al., 


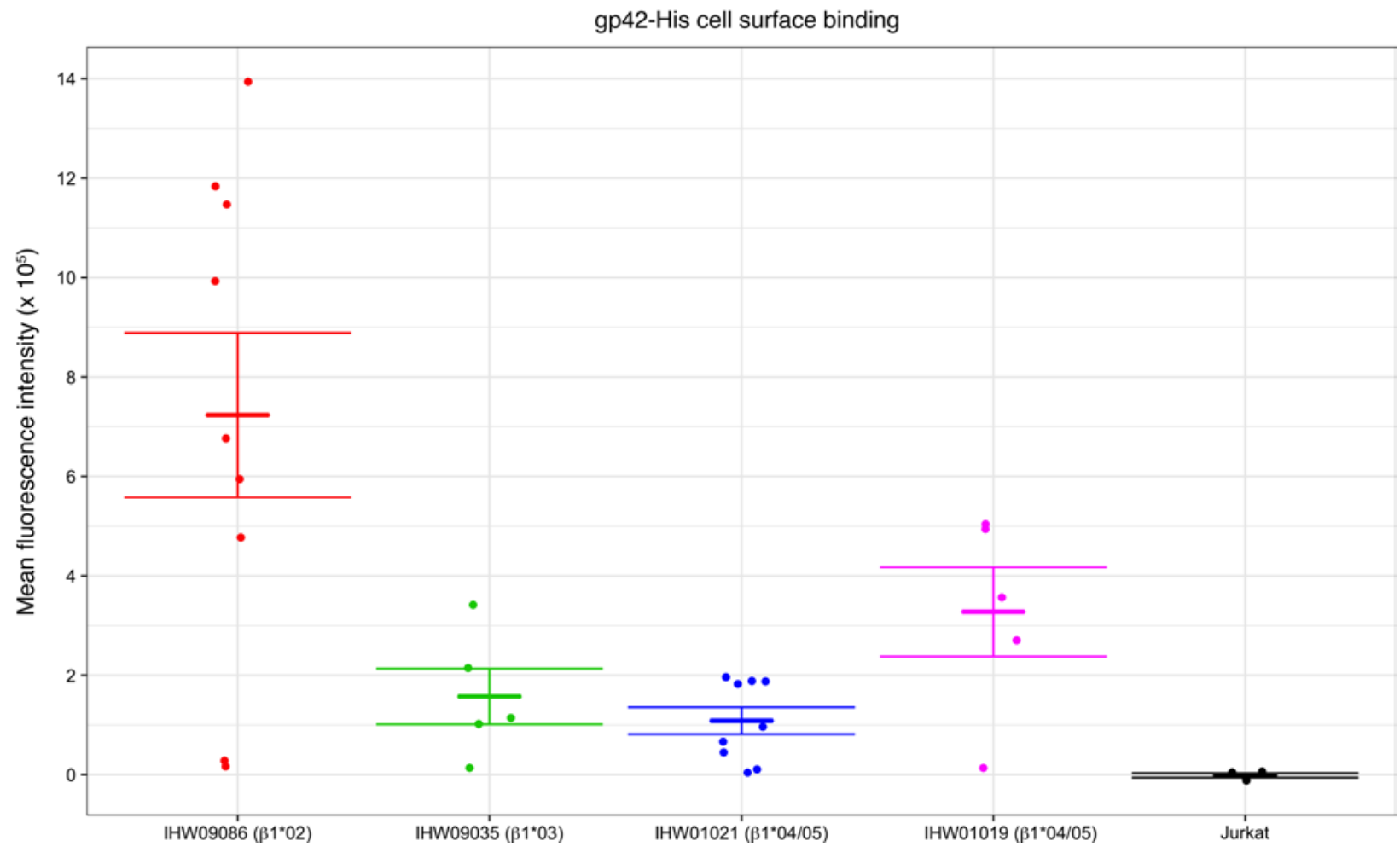

Figure 2. Lymphoblastoid cell lines expressing HLA-DQ $\beta 11^{*} 02 /{ }^{*} 02$ bind more gp42-His than cell lines expressing DQ $\beta 1{ }^{*} 03 / /^{*} 03$ or *04/*05. Cells were incubated with gp42-His on ice for 60 minutes and after washing with PBS, mouse anti-His antibody was added on ice for 60 minutes followed by Alexa488 fluorophore-conjugated anti-mouse antibody. After fixation of the cells with $2 \%$ paraformaldehyde, FACS was performed and the mean fluorescence intensity was measured. The data were obtained from 9 independent experiments. Means (short horizontal lines) \pm SEM (long horizontal lines) are shown.

unpublished observations). We then expressed the DQ $\alpha 1 / \beta 1$ heterodimers in a human LCL line, 721.174 , and infected the cells with recombinant EBV (293-EBV) that encodes a hygromycin-resistance gene (27). 721.174 cells have a deletion in HLA-DQ, -DR, and part of -DP (28). EBV infectivity was measured by real-time RT-qPCR detecting the copy number of hygromycin-resistance gene mRNA normalized against the copy number of GAPDH mRNA amplified in the same reaction as an internal control. Expression of exogenous DQ $\beta 1{ }^{*} 02 /{ }^{*} 02$ significantly enhanced EBV infection compared with ${ }^{*} 03 /{ }^{*} 03$ (Wilcoxon signed-rank $P$ value $=0.03$ ) (Figure 5). Expressing $* 03,{ }^{*} 05$, or $* 06$ slightly augmented EBV infectivity compared with vector control, although the difference was not statistically significant (Figure 5). Thus, the preference for gp 42 binding to DQ $\beta 1{ }^{*} 02 /{ }^{*} 02$ directly correlated with a similar preference for EBV infectivity in vitro. This finding provides further support for the observation that HLA class II, an EBV coreceptor, influences the rate of EBV seropositivity.

The difference in $D Q \beta 1 * 0201$ - and *0301-mediated gp 42 binding efficiency may be related to a polymorphism between the $\beta 1$ alleles that involve the gp 42 interaction interface. To further explore a mechanism for the association between HLA-DQ alleles and gp42 binding and EBV infectivity, we used the I-TASSER server (29) to predict the structures of DQ $\beta 1{ }^{*} 0201$ and ${ }^{*} 0301$. These 2 proteins share $92 \%$ identity at the amino acid level. To determine if polymorphic regions in DQ $\beta 1 * 0201$ and $* 0301$ are located at the interface between gp42 and the DQ $\beta 1$ proteins, we aligned the predicted DQ $\beta 1$ structures with the $\beta$ chain in the crystal structure of gp42 bound to HLA-DR1 (13) (PDB ID: 1KG0). Two amino acids at positions 77 and 78 in the $\mathrm{DQ} \beta 1$ proteins were found to be polymorphic at the interface (Figure 6). DQ $\beta 1{ }^{*} 0201$ has glycine and glutamine at these positions, respectively, while ${ }^{*} 0301$ has glutamine and valine. Therefore, the polymorphism in the region may contribute to the disparity in gp42 binding capacity.

Type 1 and type $2 E B V$ gp 42 bind to HLA-DQ with similar efficiency; however, a neutralizing monoclonal antibody against gp 42 blocks type 1 binding more efficiently than type 2. Two EBV types circulate in most populations: type 1 and type 2 . Type $1 \mathrm{EBV}$ is predominantly prevalent in the developed world, whereas type $2 \mathrm{EBV}$ is present in people in equatorial Africa and New Guinea (3). Type 1 gp42 and type 2 


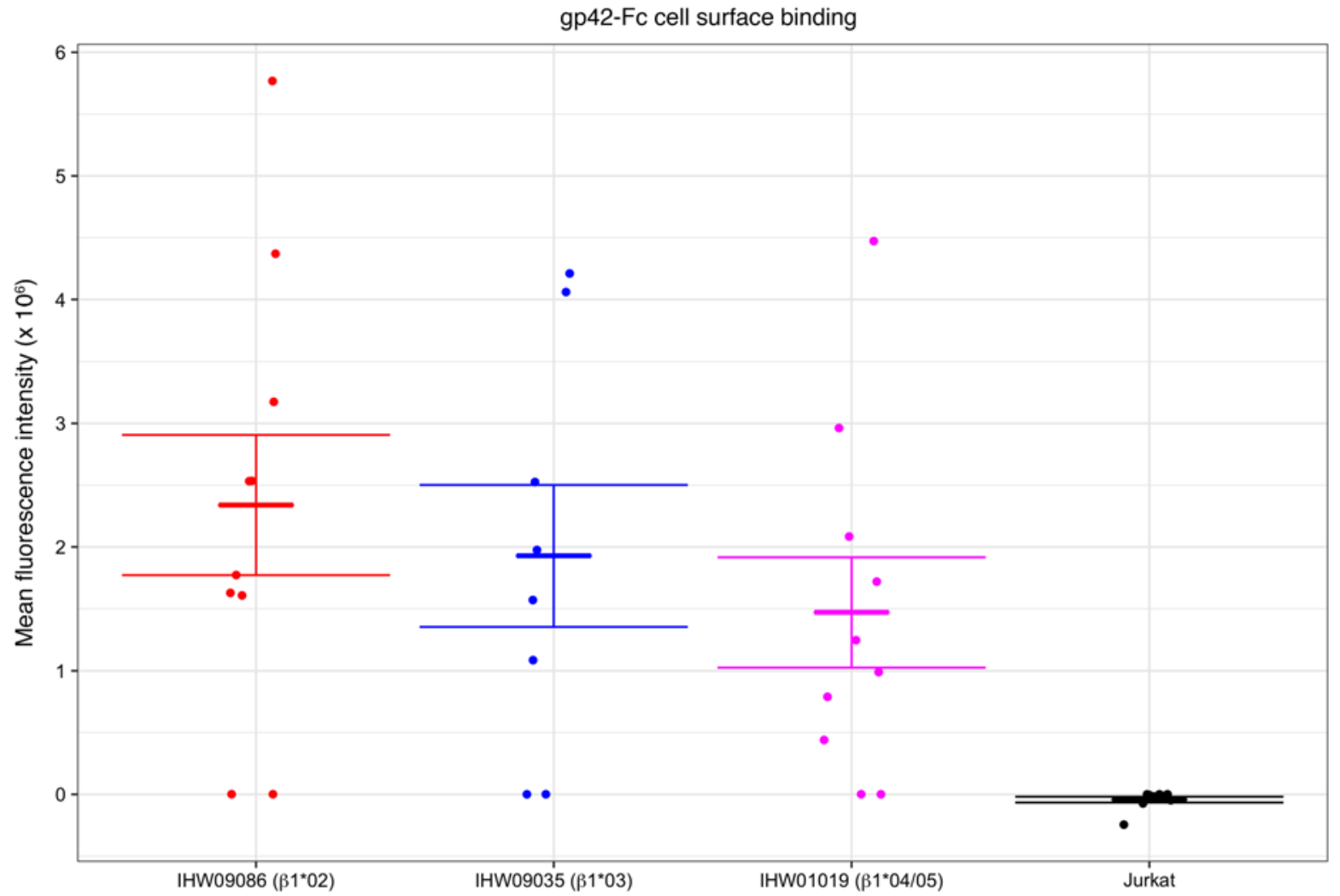

Figure 3. Lymphoblastoid cell lines expressing DQ $\beta 1{ }^{*} 02 /{ }^{*} 02$ bind more dimerized gp42 than cells expressing $D Q \beta 1 * 03 /{ }^{*} 03$ or ${ }^{*} 04 /{ }^{*} 05$ alleles. Cells were incubated with purified gp42-Fc as described in the legend to Figure 2 for gp42-His protein. Human TruStain FcX Fc receptor blocking solution (Biolegend) was then added to the cells for 5 minutes before adding anti-human IgC Alexa488 antibody, and gp42 binding was quantified by FACS. The cells were also incubated with IgG-Fc and binding of Ig-Fc was measured. The net mean fluorescence intensity of gp42-Fc binding was calculated by subtracting the background binding from IgG-Fc. The data were derived from 10 independent experiments. Means (short horizontal lines) \pm SEM (long horizontal lines) are shown.

gp42 share 98\% amino acid identity. All 5 amino acids that differ between type 1 and type 2 gp42 are located in the C-type lectin domain of gp42 that is important for HLA class II interaction (13, 30). To identify if any of the polymorphic regions are located near the HLA-DQ-binding interface, we used the I-TASSER server (29) to predict the structure of type 2 gp42. For this analysis we used the crystal structure of type 1 gp42 bound to HLA-DR1 (13) (PDB ID: 1 KG0) as a template. The predicted structure identified a polymorphic region between types 1 and 2 gp42 near the HLA-DQ-binding interface (Figure 7). Therefore, we determined if there was a difference between the 2 types of gp 42 in HLA binding. Using human LCLs and transfected mouse cells that express HLA-DQ in the absence of HLA-DR or -DP, we found that types 1 and 2 EBV gp42 bound to HLA class II in a similar manner (Figure 8). A monoclonal antibody against gp42, F-2-1, potently blocks EBV infection of B cells but not epithelial cells $(7,8)$. Since epithelial cells usually do not express significant amounts of HLA class II, we postulated that the F-2-1 epitope might be located at the interface where gp42 and HLA class II

Table 5. HLA class II genotypes of lymphoblastoid cell lines used in EBV gp42 protein-binding assays

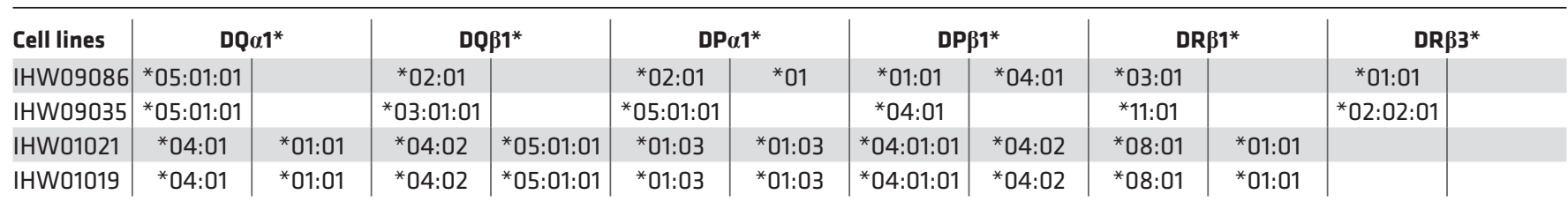




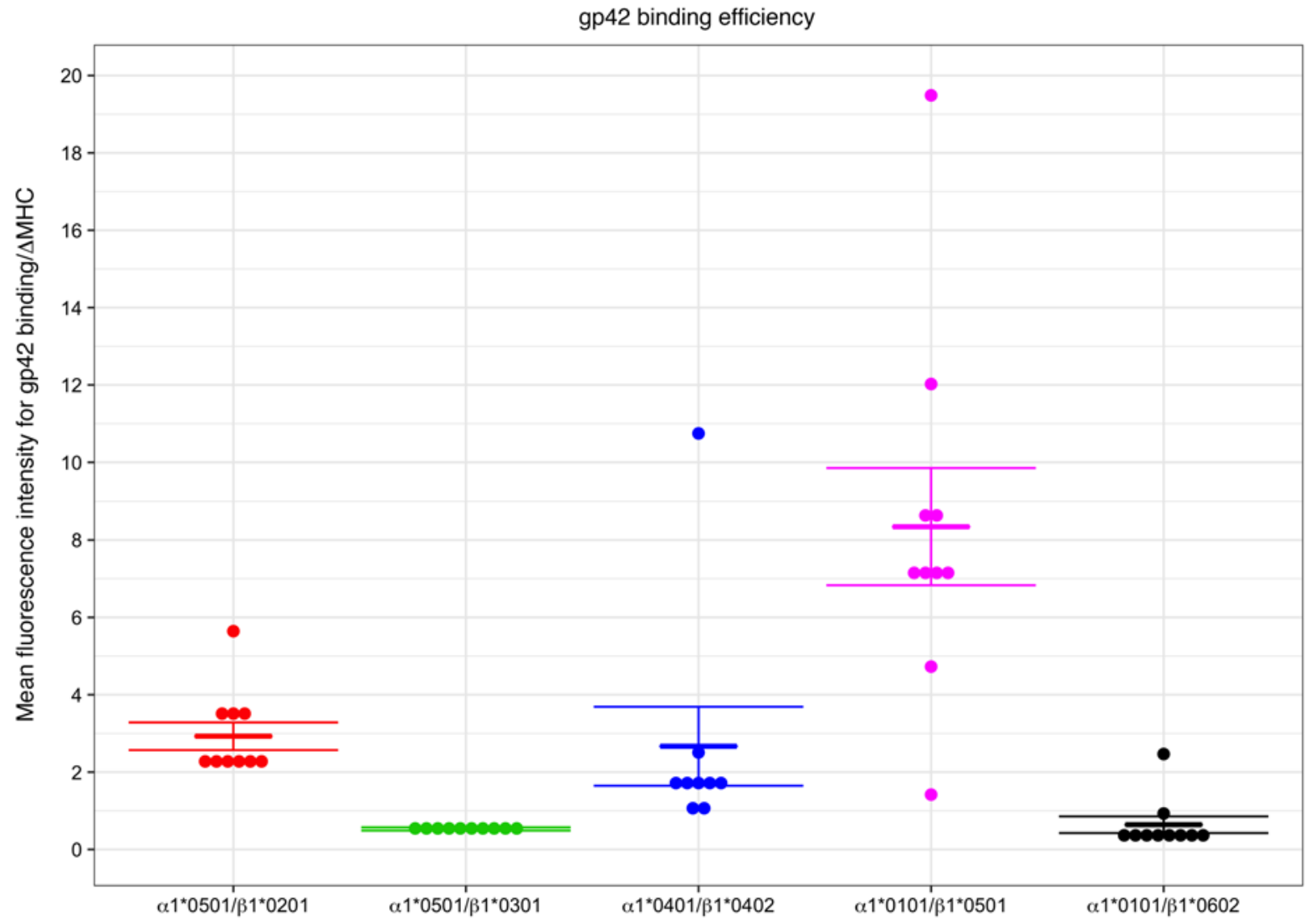

Figure 4. Cells expressing HLA-DQ $\beta 1 * 02 / * 02$ bind more gp42 than cells expressing $D Q * 03 /{ }^{*} 03$ or $* 06 / * 06$ when expressed independently of other HLA molecules. Mouse 3T3 cells expressing HLA-DQ $\alpha \beta$ heterodimers were incubated with purified gp 42-His followed by anti-His antibody as described in the legend to Figure 2 . The amount of gp42 bound was quantified by FACS. Cell surface HLA-DQ $\beta 1$ levels were also quantified in the same experiment by staining with la3 antibody followed by isotype-specific secondary antibody (Alexa647). The ratio of the mean fluorescence intensity (MFI) for gp42 binding (FL-4) divided by $\triangle \mathrm{MHC}$, which is the ratio of cell surface DQ expression to internal control GFP (24), is shown. The results were the same when we defined gp42 binding efficiency as the ratio of the MFI of gp42 binding divided by the MFI of HLA-DQ. The data were obtained from 10 independent experiments. Means (short horizontal lines) \pm SEM (long horizontal lines) are shown.

interact. To test this possibility, we performed gp 42 binding assays using human LCLs in the presence of F-2-1 or isotype control antibody. Monoclonal antibody F-2-1 blocked about $80 \%$ of type 1 EBV gp42 binding to HLA class II DQ $\beta 1 * 0201$ and $85 \%$ of type 1 gp42 binding to DQ $\beta 1 * 0301$ (Figure 9A). However, F-2-1 blocked only about $35 \%$ of type 2 gp 42 binding to $\beta 1 * 0201$ and $50 \%$ binding to $\beta 1 * 0301$ (Figure 9B) $(P<0.001$ for type 1 vs. type 2 gp 42 binding). These results suggest that although the polymorphism in type 2 gp 42 still allows efficient HLA class II binding, the interaction interface might be partially excluded from the F-2-1 blocking epitope.

Table 6. Stability of HLA-DQ on mouse 3T3 cells expressing DQ $\alpha \beta$ heterodimers measured by $\Delta \mathrm{MHC}^{\mathrm{A}}$ and normalized against $\alpha 1$ *01:01/ק1*06:02

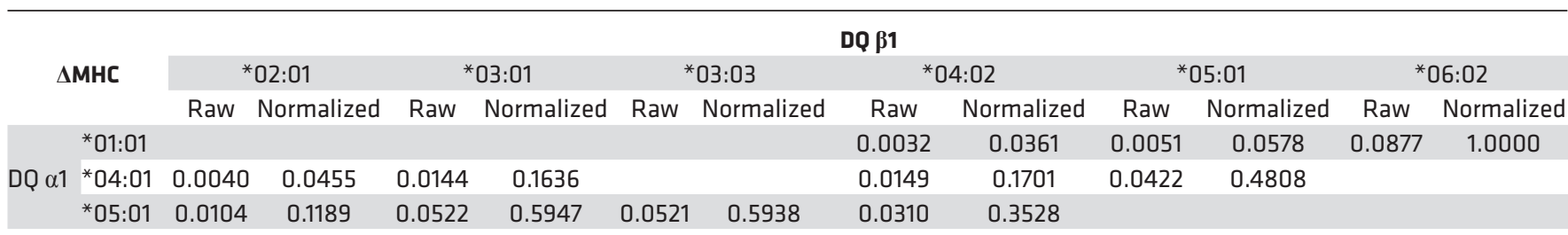

${ }^{\mathrm{A}} \triangle \mathrm{MHC}$ was calculated as a ratio of cell surface $\mathrm{DQ}$ expression level to the internal control GFP of the cell population. 


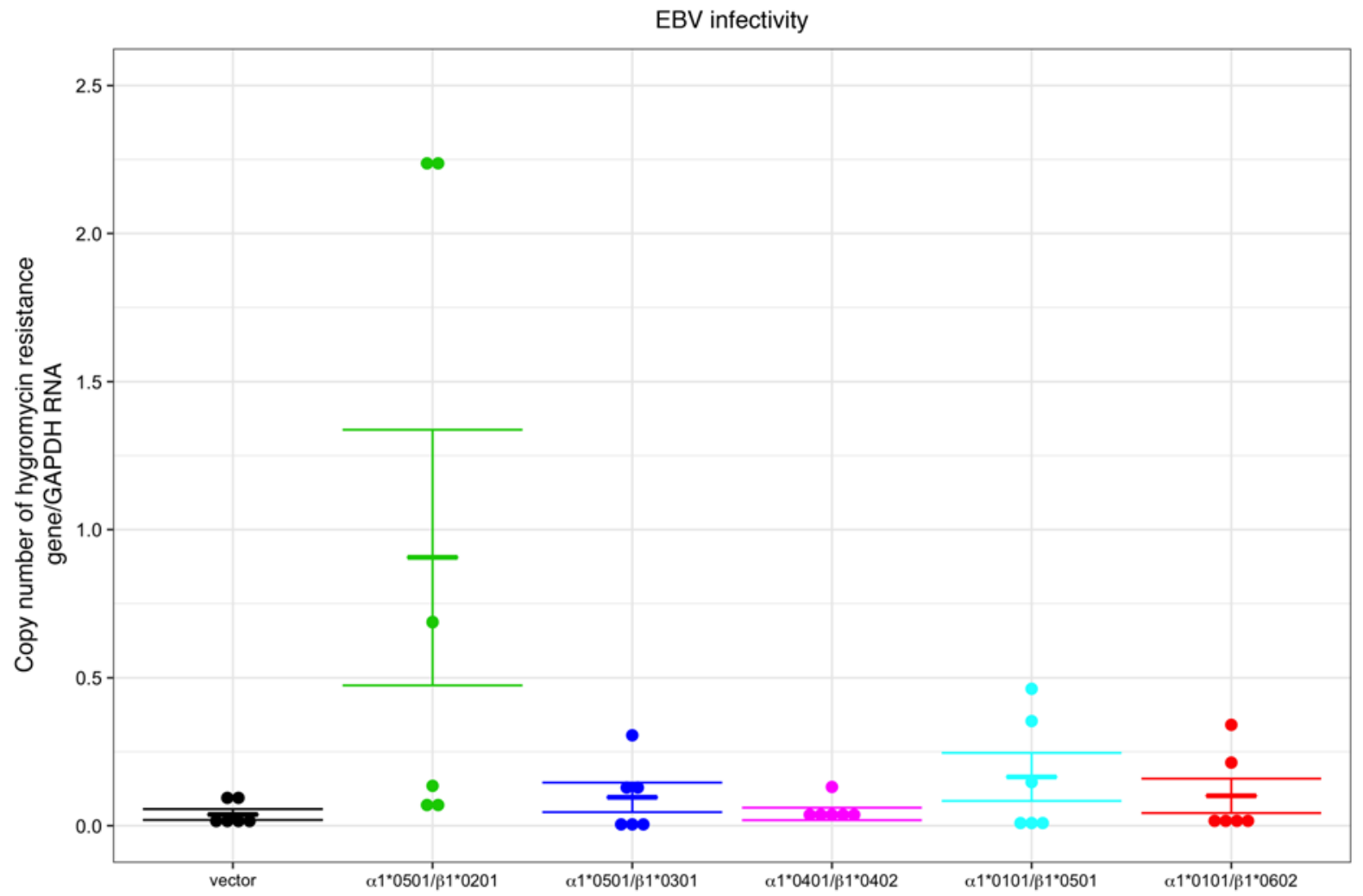

Figure 5. HLA-DQ $\beta 1^{*} 02 /^{*} 02$ alleles facilitate EBV infectivity more efficiently than other alleles. Human 721.174 cells were nucleofected with plasmids encoding DQ $\alpha 1$ and $\beta 1$ alleles and infected with 293-EBV 48 hours later. RNA extraction was performed 3 days after infection, and copy numbers for the products of the hygromycin-resistance gene and GAPDH (internal control) were determined by real-time RT-qPCR in duplicate. The data were derived from 6 independent experiments. Means (short horizontal lines) \pm SEM (long horizontal lines) are shown.

\section{Discussion}

While EBV is one of the most ubiquitous viruses in the human population, about $5 \%$ of adults worldwide are not infected with EBV. A number of factors may be responsible for resistance to infection, including socioeconomic factors that may affect exposure to the virus and genetics of the individual (18-21). In this study, we compared a cohort of 106 EBV-seronegative and 218 -seropositive blood donors screened from $\sim 3,300$ individuals and found that persons who were positive for HLA-DQ $\beta 1 * 04 /{ }^{*} 05$ or $-\mathrm{DQ} \beta 1{ }^{*} 06 /{ }^{*} 06$ were more likely to be EBV seronegative compared with other DQ $\beta 1$ combinations, while those who were positive for DQ $\beta 1{ }^{*} 02 /{ }^{*} 02$ were more likely to be EBV seropositive. However, with 15 different possible combinations of DQ $\beta 1$ genotypes and a relatively small sample size due to the low rate of EBV seronegativity in adults, it is not surprising that while initial $P$ values indicated significant associations between EBV serostatus and certain HLA-DQ $\beta 1$ alleles, after adjusting for multiple comparisons the associations did not remain significant. While this is the largest study thus far testing HLA associations with EBV infectivity in seronegative persons, our results indicate the need for an even bigger sample size, since HLA-DQ is highly polymorphic and allele frequencies vary in the general population. Although the blood donor studies did not reach statistical significance, the results are supported by our in vitro experiments showing that EBV gp42 bound weakly to cells expressing HLA-DQ $\beta 1$ ${ }^{*} 04 /{ }^{*} 05$ and ${ }^{*} 06 /{ }^{*} 06$, compared with cells expressing DQ $\beta 1{ }^{*} 02 /{ }^{*} 02$. While EBV proteins interact with hundreds of

Table 7. Significance of different HLA-DQ $\beta 1$ alleles in binding to EBV gp42

\begin{tabular}{|c|c|c|c|c|}
\hline \multirow{2}{*}{$P$ value $^{A}$} & \multicolumn{4}{|c|}{$D Q \beta 1^{*}$} \\
\hline & ${ }^{*} 03$ & ${ }^{*} 04$ & ${ }^{*} 05$ & ${ }^{*} 06$ \\
\hline${ }^{*} 02$ & $<0.001$ & 0.016 & 0.004 & 0.001 \\
\hline *03 & & $<0.001$ & $<0.001$ & 0.353 \\
\hline UQ $\mid{ }^{*} 04$ & & & 0.01 & 0.001 \\
\hline${ }^{*} 05$ & & & & $<0.001$ \\
\hline
\end{tabular}

${ }^{A}$ Data derived from 8 independent experiments shown in Figure 4. 


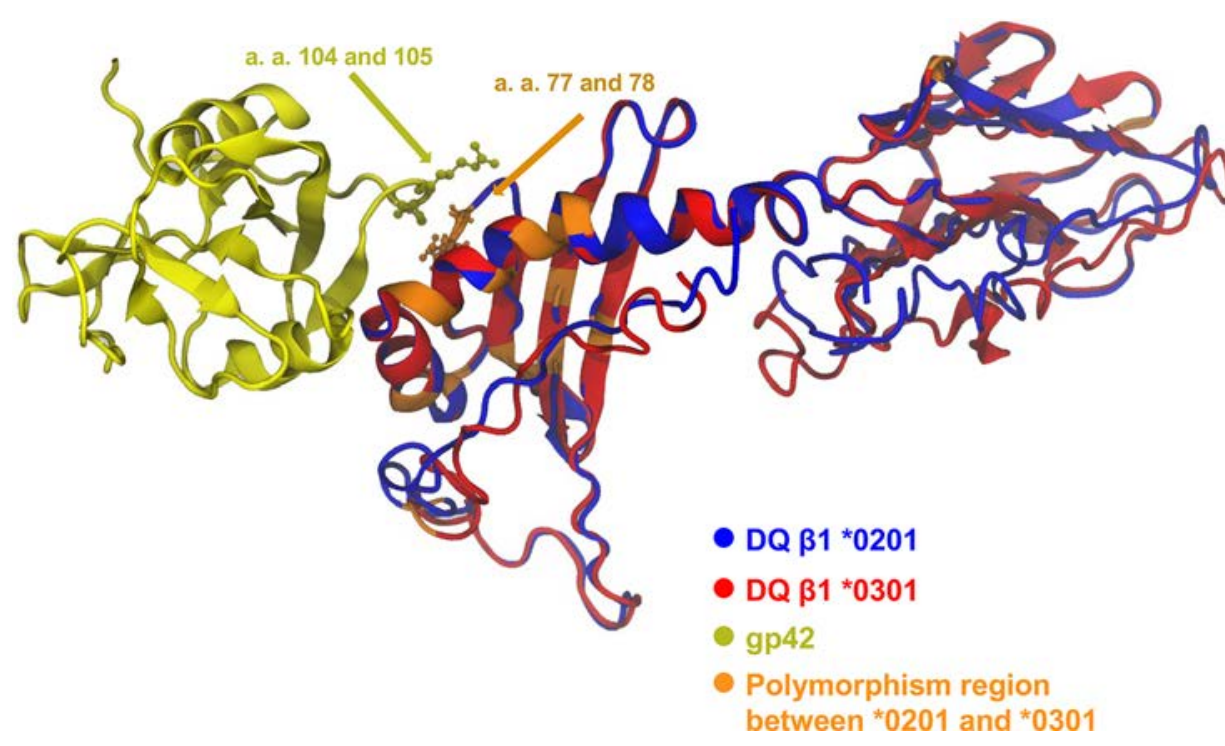

Figure 6. Predicted structures of DQ $\beta 1$ *0201 (blue) and DQ $\beta 1{ }^{*} 0301$ (red). The polymorphic regions between the $2 \mathrm{DQ} \beta 1$ alleles are shown in orange. The interface between DQ $\beta 1{ }^{*} 0201 /{ }^{*} 0301$ and gp42 (yellow) was modeled by aligning the predicted structures with the $\beta$ chain of the crystal structure of gp 42 bound to HLA-DR1 (13). Amino acids 77 and 78 in DQ $\beta 1$ are Gly and Glu in $\beta 1{ }^{*} 0201$, respectively, and are Glu and Val in ${ }^{*} 0301$, respectively; these 2 amino acids interact with amino acids 104 (Thr) and 105 (Arg) of EBV gp42.

host cell proteins, which may contribute to virus infectivity at various steps including virus entry, replication, and immune control $(3,31,32)$, the observation that the interaction of gp42 with HLA class II is essential for EBV

infection $(7,9,12,33)$ may explain the ability of a single genetic locus to influence infectivity with EBV.

Other much smaller studies have looked for associations between MHC class II alleles with EBV infectivity in seronegative persons. Jabs et al. reported a significant association between EBV seronegativity and HLA-DR 13 in a cohort of 20 EBV-seronegative and 32 EBV-seropositive healthy donors (34). Since HLA-DR 13 is genetically linked to HLA-DQ6 (DQ $\beta 1 * 06$ ), especially in populations of European descent (35), it is possible that DQ $\beta 1 * 06$ might have contributed to EBV seronegativity as an HLA-DR $\beta 1 * 13-\mathrm{DQ}$ $\beta 1 * 06$ haplotype in their cohort. In a study of 106 pediatric allograft recipients, Hocker and colleagues identified HLA-DR7 as an independent risk factor for EBV infection (36). Since HLA-DR7 is genetically associated with DQ $\beta 1{ }^{*} 02 /{ }^{*} 02$, these results are consistent with our observation of a higher than expected proportion of HLA-DQ $\beta 1 * 02$ subjects that are seropositive.

MHC class I alleles have also been shown to correlate with EBV infectivity in small studies. Durovic et al. showed that EBV seronegativity among individuals older than 60 years is associated with HLA$\mathrm{C}$ and HLA-Bw4 variants in a cohort of 17 EBV-seronegative and $39 \mathrm{EBV}$-seropositive subjects (37). Edmonds and colleagues found that HLA A10, A29, and B15 were underrepresented in a cohort of 27 EBV-seronegative persons when compared with 95 seropositive persons (38). Boyer et al. studied 55 EBVseronegative and 367 EBV-seropositive persons and found an association of a blank HLA A locus with EBV seronegativity (39).

Previous studies have shown that in addition to binding HLA-DQ, gp42 also binds to DR and DP $(9,12)$. Therefore, DR and DP are also likely to be important in mediating EBV infection. Certain combinations of $\mathrm{DQ}, \mathrm{DR}$, and DP alleles may allow a small fraction of the general population to be refractory to infection by EBV. The HLA class II locus has at least 4,000 alleles with tens of thousands of possible combinations (IPD-

Figure 7. Predicted structures of EBV type 2 gp42 (yellow) superimposed on the crystal structure of type $1 \mathrm{gp} 42$ (gray) bound to DQ $\beta 1$ (red) and DQ $\alpha 1$ (blue) (13). Amino acids 113 and 114 near the $\mathrm{DQ} \beta 1$-binding interface in type 1 gp42 are Gly and Glu, respectively, and are Clu and Arg in type 2 gp 42 , respectively.

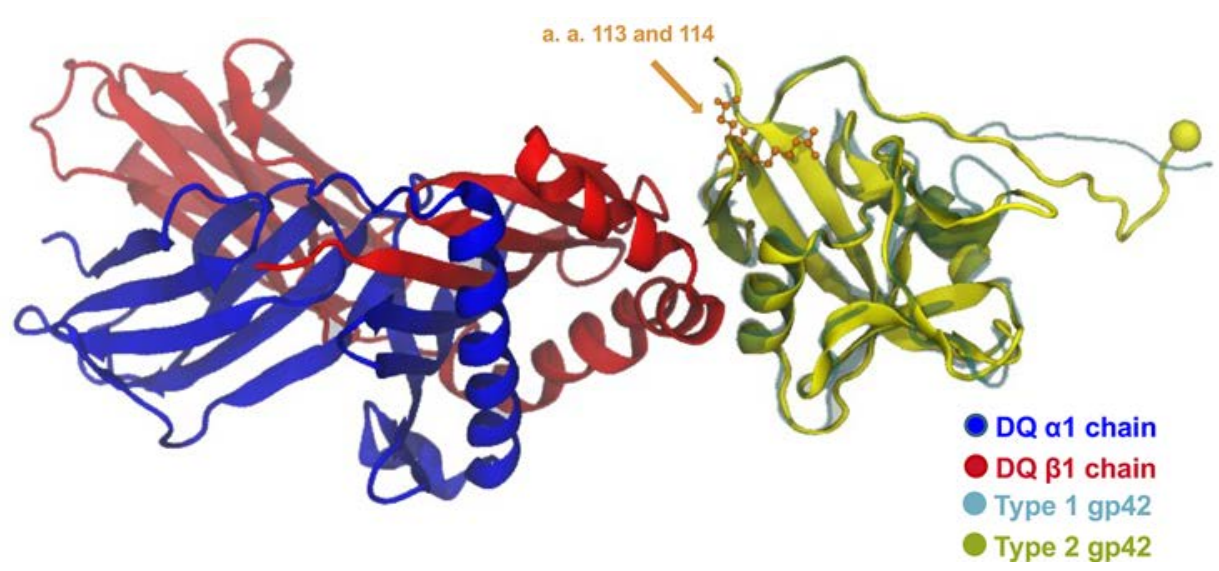


Type 1 gp42 binding efficiency

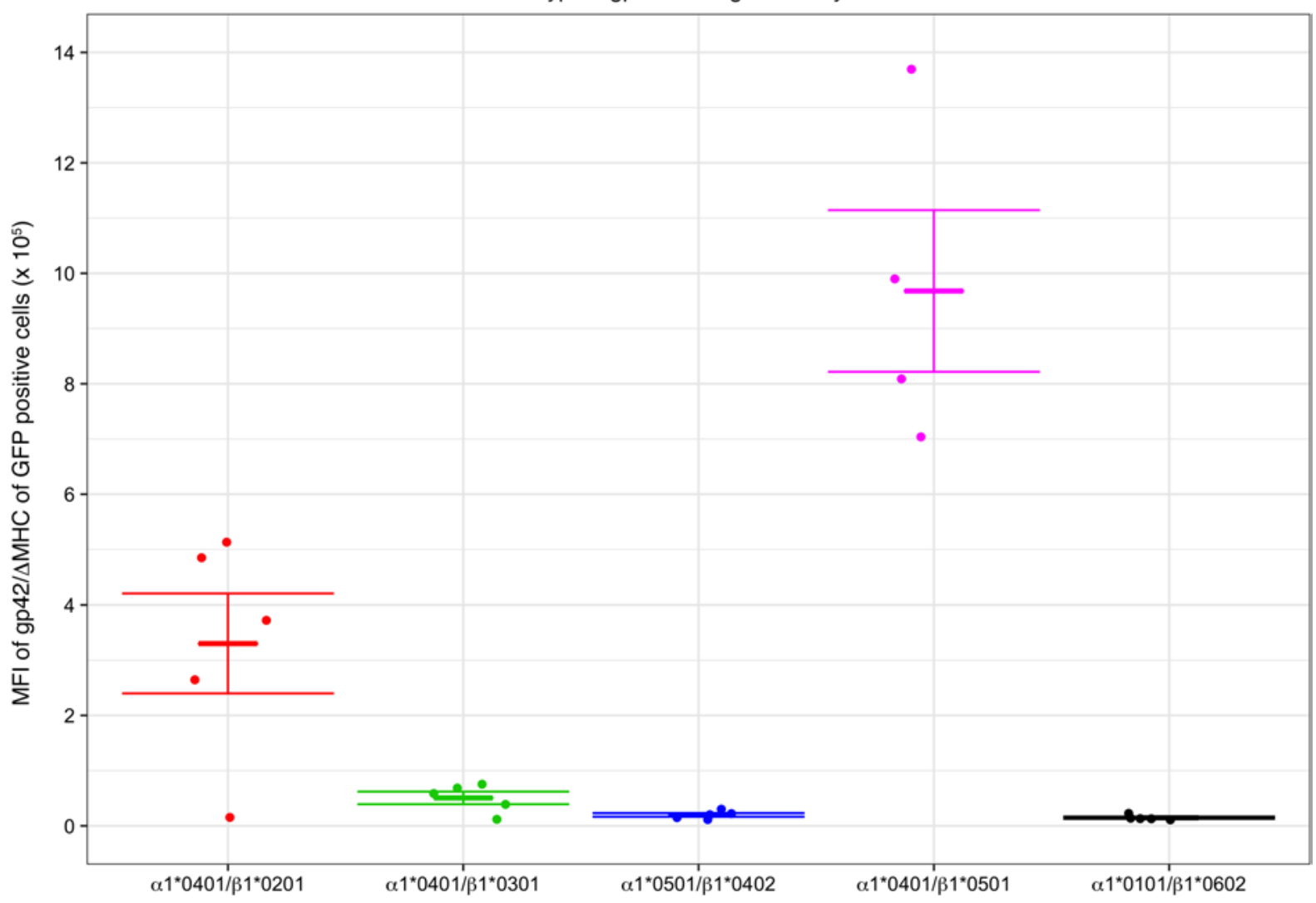

Type 2 gp42 binding efficiency

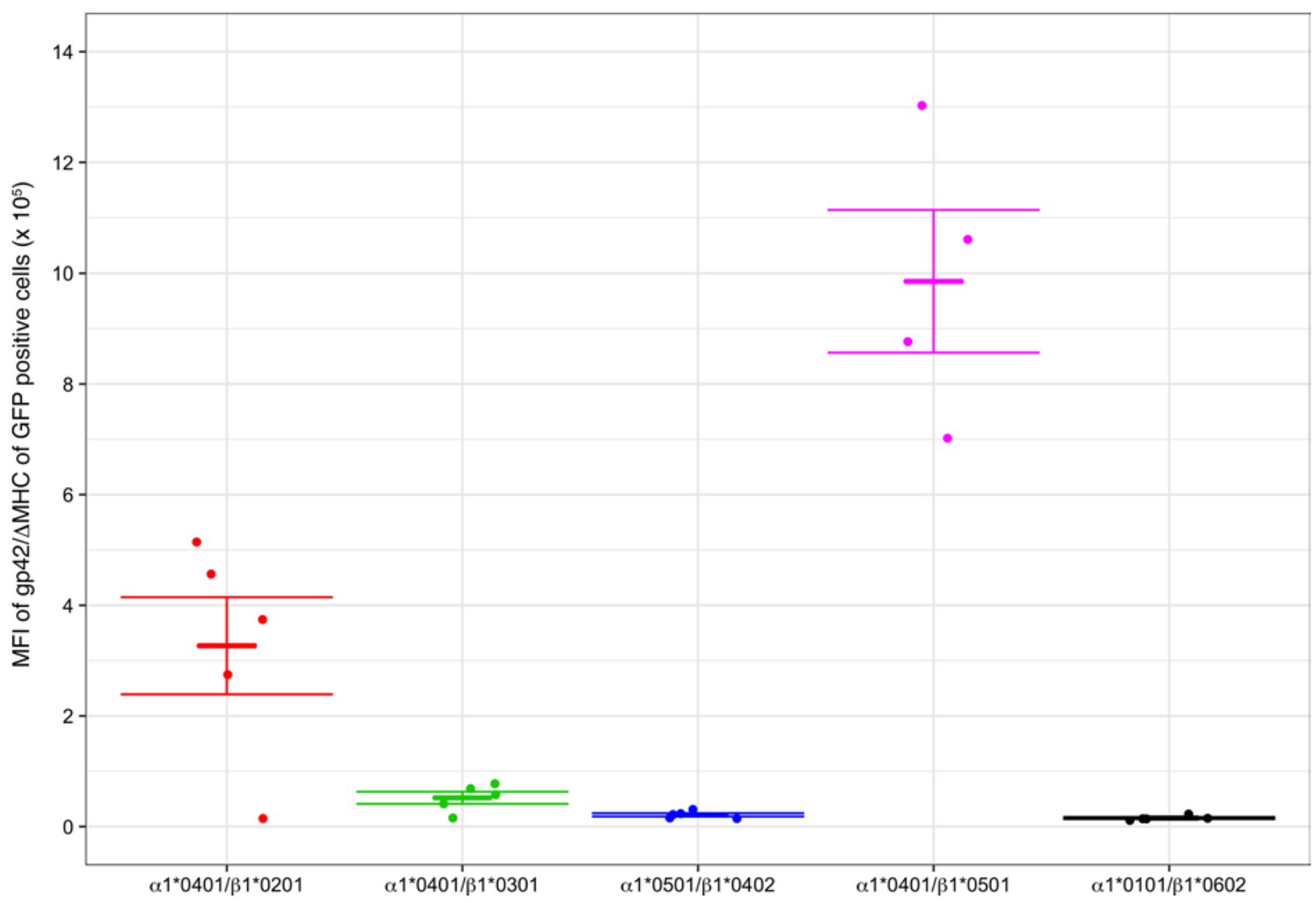


Figure 8. Type 1 and type 2 EBV gp42 bind to HLA-DQ with similar efficiency. Mouse 3T3 cells expressing HLA-DQ $\alpha \beta$ heterodimers were incubated with the same amount of purified type 1 (top) or type 2 (bottom) gp42-His protein followed by anti-His antibody staining and detection of gp42 by FACS as described in the legend to Figure 4. MFI, mean fluorescence intensity; $\triangle \mathrm{MHC}$, the ratio of cell surface DQ expression to internal control GFP (24). The data were obtained from 5 independent experiments. Means (short horizontal lines) \pm SEM (long horizontal lines) are shown.

IMGT/HLA database: https://www.ebi.ac.uk/ipd/imgt/hla) (40); therefore, it is virtually impossible to dissect the roles of DQ, DR, and DP together. Accordingly, we focused our study on DQ alleles without including the role of DR and DP. Haan and Longnecker expressed HLA-DQ heterodimer $\alpha 1^{*} 03: 01 / \beta 1 * 02: 01$ and $\alpha 1^{*}$ 05:01/ $\beta 1 * 03: 03: 02$ in human LCLs that do not express endogenous HLA-DR, -DP, or -DQ. Using recombinant EBV that encodes GFP, they showed that expression of DQ $\beta 1{ }^{*} 02: 01$ increased EBV infectivity by 20-fold or more, while expression of $\beta 1^{*} 03: 03$ did not increase EBV infectivity (17). Additional studies by these authors showed that EBV gp42 interacts with the N-terminal region of DQ $\beta 1(13,23)$. We expanded these observations by expressing DQ $\beta 1{ }^{*} 04,{ }^{*} 05$ and ${ }^{*} 06$, in addition to ${ }^{*} 02$ and ${ }^{*} 03$. Our findings that DQ $\beta 1 * 02$ not only bound significantly more gp 42 than DQ $\beta 1 * 03$ or other alleles in human cells, but also facilitated significantly more infection, are consistent with the previous findings. This provides a mechanism that may explain the observation that a higher than expected proportion of EBV seropositive individuals have HLA-DQ $\beta 1 * 02$ alleles.

The evolution of diversity of HLA molecules is thought to be the result of selection and adaption from combating infection (16). Paradoxically, EBV has adapted to use MHC class II molecules as entry coreceptors through its interaction with gp42. This interaction is essential for EBV infection (33). Although gp42 binding to HLA involves only the $\beta 1$ chain outside the peptide groove of the $\alpha \beta$ heterodimer $(9,10,13)$, gp42 binding also interferes with the interaction of HLA-DR with the T cell receptor, inhibits generation of cytotoxic $\mathrm{T}$ cells, and impairs antigen presentation $(9,14,15)$. Therefore, gp42 may have evolved multiple functions so that while it is essential for EBV infection, it may inhibit the cellular immune response to the virus.

\section{Methods}

Sample collection and EBV serology testing. Sera from about 3,300 healthy blood donors were collected over a 15 -year period in the United States and Switzerland. Blood was obtained from blood donors at the Warren G. Magnuson Clinical Center at the NIH ( 2,400 individuals), the Research Donor Program at the Frederick National Laboratory for Cancer Research (392 donors), and the Blood Transfusion Center in Basel, Switzerland (515 donors; see ref. 37). Sera were tested for IgG antibodies to the EBV viral capsid antigen using Captia Epstein-Barr Viral Capsid Antigen IgG ELISA kits following the manufacturer's instructions (Trinity Biotech USA Inc.) or by multiplex microparticle technology (Luminex 200 Technology, Luminex Corp.) (37). All EBV-negative samples were then tested independently again at the NIH Department of Laboratory Medicine by a technician who was blinded to the prior results to confirm that each of these individuals was EBV seronegative.

Peripheral blood mononuclear cells (PBMCs) were isolated from buffy coat preparations of human blood using Ficoll-Paque Plus (GE Healthcare BioSciences). LCLs were established by infecting PBMCs with EBV B95.8 and propagated as described below.

Cells and reagents. LCLs were obtained from the International Histocompatibility Working Group (Fred Hutchinson Cancer Research Center) and maintained in RPMI 1640 medium with 15\% FBS. Mouse 3T3 cells were grown in Dulbecco's Modified Eagle Medium with 10\% FBS. Anti-CD21 (R\&D Systems, catalog MAB4909), mouse anti-His (GE Healthcare Life Sciences, catalog 27-4710-01), rabbit anti-His (Cell Signaling Technology, catalog 2365), and anti-HLA-DQ (MP Biomedicals, clone Ia3, catalog 68571) were used to stain cells. Secondary antibodies (anti-mouse IgG-Alexa488, catalog A11001; anti-mouse IgG2aAlexa647, catalog 407116; anti-rabbit IgG-Alexa488, catalog A11034; and anti-human IgG-Alexa488 catalog A11013) were all purchased from Life Technologies. Human TruStain FcX Fc receptor blocking solution (catalog 422301) was obtained from Biolegend. F-2-1 antibody against EBV gp42 was previously described (7).

Generation of gp42-Fc, and type 1 and type 2 gp42-His proteins. gp42-Fc fusion protein (type 1) was made by transfecting CV1/EBNA1 cells with plasmid pDC409-BZLF2 purified with a protein A affinity column as described previously (9). Soluble gp42-His (type 1) was produced by PCR amplification of codon-optimized BZLF2 (the gene that encodes EBV gp42) from EBV strain B95.8 using a forward primer encoding a His-tag 


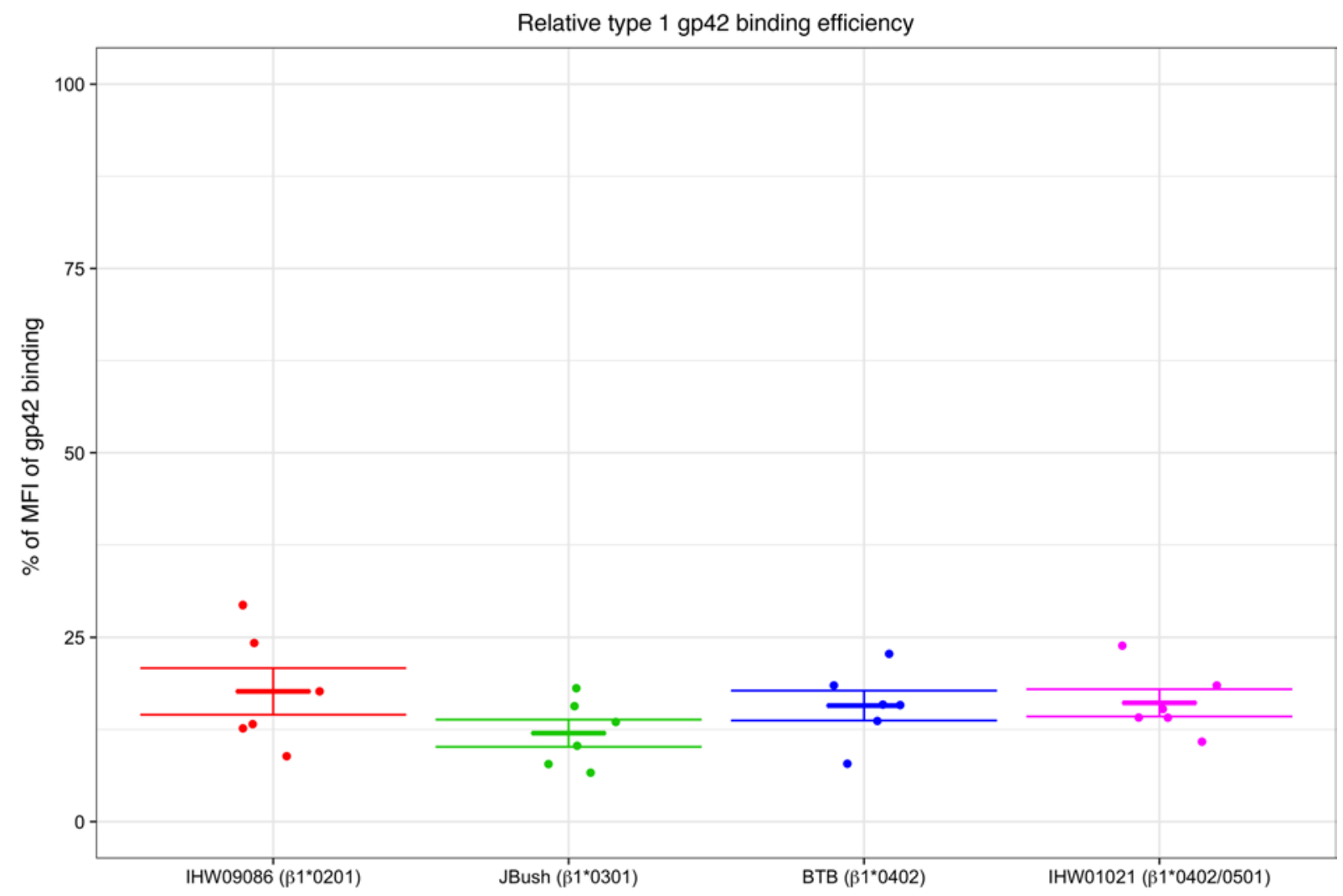

Relative type 2 gp42 binding efficiency

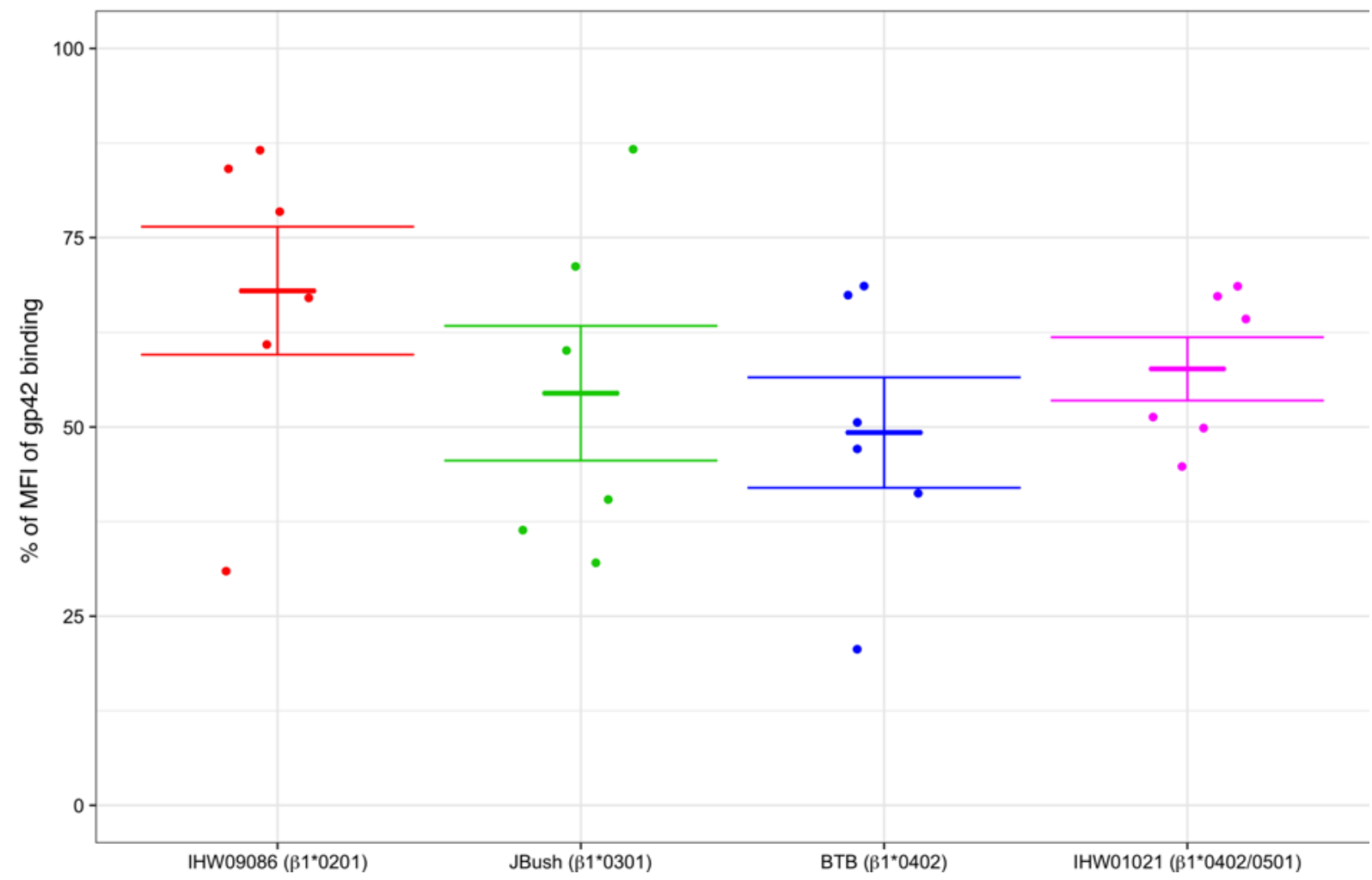

Figure 9. A neutralizing monoclonal antibody against gp42 blocks type 1 gp42 binding more efficiently than type 2 gp42 binding to human cells expressing different HLA class II alleles. Type 1 (top) or type 2 (bottom) gp42 proteins were preincubated with monoclonal anti-gp42 Ab, F-2-1, or a control antibody at room temperature for 30 minutes, and added to human lymphoblastoid cell lines and binding was assayed as described in the legend to Figure 2 except that detection of His-tagged gp42 was performed using rabbit anti-His antibody (Cell Signaling Technology) followed by Alexa488-conjugated 
anti-rabbit secondary antibody. The relative percentage of gp42 binding to cells in the presence of F-2-1 antibody was calculated against that of the control antibody, which was set at $100 \%$. The relative binding of gp42 in the presence of F-2-1 antibody is shown. MFI, mean fluorescence intensity. The data were derived from 6 independent experiments. Means (short horizontal lines) \pm SEM (long horizontal lines) are shown.

(underlined) 5'-GGGCGTGCTAGCCCACCACCACCACCACCACGGAGGAAGAGTGGCCGC-3' and a reverse primer 5'-CCCGGATCCTCAGGAGTTGCTTCTCTGAGC-3', digested with XbaI and BamHI and subcloned into the corresponding restriction sites of plasmid CMV/R 8kb (VRC 8405) containing a human CD5 signal sequence (41) to yield plasmid CMV/R 8кb-gp42. Expi293F cells (Life Technologies) were transfected with plasmid CMV/R 8kb-gp42, and gp42-His was purified from the supernatant of the cells using Talon metal affinity resin (Clontech) (42) followed by size-exclusion chromatography using Superose 6 10/300 GL (GE Healthcare Life Sciences). Type 2 gp42-Fc fusion protein was constructed using the sequence from EBV AG876 strain (GenBank: DQ279927.1) by gene synthesis (GenScript) and cloned into the backbone of the CMV/R 8kb-gp42 plasmid. Soluble protein was expressed and purified as described for type 1 gp42-His.

Genomic DNA extraction. Genomic DNA was extracted from PBMCs or blood clots using DNeasy Blood \& Tissue Kits (Qiagen) following the manufacturer's instructions. For DNA extraction from blood clots, the samples were first homogenized in $5 \mathrm{ml} \mathrm{ACK}$ lysis buffer (Lonza) and digested with proteinase $\mathrm{K}$ at $56^{\circ} \mathrm{C}$ overnight before using the DNeasy Blood \& Tissue Kit.

HLA typing. Intermediate/high-resolution typing for HLA class II loci was performed using LABType sequence-specific oligonucleotide (SSO) Typing Kits obtained from One Lambda, Inc. LABType uses the reverse SSO DNA typing method with Luminex 200 Technology. First, target DNA was amplified by PCR using a group-specific primer. The PCR product was biotinylated during the amplification process, which allows it to be detected using R-phycoerythrin-conjugated streptavidin (SAPE). Some samples required the use of sequence-based typing (SBT) in order to verify or further identify the HLA type to a high-resolution level. SBT was performed utilizing a big dye terminator chemistry method. The primary PCR amplification reaction produced a $1.5-\mathrm{kb}$ amplicon. Reagents for primary amplification and sequencing were obtained from HLA Sequence-Based Typing Kits (Life Technologies). The primary-amplification PCR products were purified from excess primers, dNTPs, and genomic DNA using ExoSAP-IT (American Life Science). Each template was sequenced in the forward and reverse sequence orientation. Excess dye terminators were removed from the sequencing products using ethanol precipitation. The reaction products were reconstituted with $15 \mu 1$ of Hi-DiTM Formamide (PE Applied Biosystems/PerkinElmer) and analyzed on an Applied Biosystems (ABI) Prism* 3730xL DNA Analyzer.

Generation of mouse cell lines stably expressing human HLA-DQ alleles. Stable cell lines expressing HLADQ alleles were generated as previously described (24). Briefly, recombinant retroviruses encoding various $\mathrm{DQ} \alpha 1$ and $\beta 1$ alleles were generated by transfecting expression plasmids (in a pMXs-IG backbone) into Plat-E packaging cells (Cell Biolabs). Mouse 3T3 cells were then transduced with the resulting pMXs-IG-DQ $\beta 1$ retroviruses encoding different $\beta 1$ alleles and a puromycin-resistance gene. Stably transfected cells were subsequently transduced with pMXs-IG-DQ $\alpha 1$ retroviruses carrying different HLA-DQ $\alpha 1$ genes and a GFP gene. FACS was carried out to enrich the GFP-positive cell population.

Generation and expression of HLA DQ plasmids for expression in human cells. DQ $\alpha 1$ chain *01:01:01, ${ }^{*} 04: 01: 01$, and ${ }^{*} 05: 01: 01: 01$, and DQ $\beta 1$ chain ${ }^{*} 02: 01: 01,{ }^{*} 03: 01: 01,{ }^{*} 04: 02: 01,{ }^{*} 05: 01: 01: 01$, and *06:02:01 (dbMHC database, NCBI) were subcloned into pcDNA3.1. The resulting plasmids were verified by sequencing and transfected into LCL 721.174 cells by nucleofection using a Nucleofector with Kit V (Lonza, catalog VCA-1003).

EBV infection and detection. 293-EBV, a recombinant EBV encoding a hygromycin-resistance gene, was prepared as previously described (27). Cells infected with 293-EBV were harvested and total RNA was extracted using an RNeasy Plus Kit (Qiagen). To eliminate DNA contamination, the RNA was applied to a column that removes DNA and also treated with DNaseI (Roche) at $1.0 \mathrm{U} / \mu \mathrm{l}$ at room temperature for 15 minutes and repurified. Quantitative real-time RT-PCR was performed using the One-step RT-PCR Master mix (Promega) with a 7500 Real-Time PCR machine. Primers (5'-CTATCAGAGCTTGGTTGACGG-3' and 5'-CTTCTACACAGCCATCGGTC-3') and a FAM-labeled probe (5'-ACACAAATCGCCCGCAGAAGC-3') for detection of the hygromycin-resistance gene product were synthesized by Integrated DNA Technologies. Primers and a VIC-labeled probe set detecting GAPDH was purchased from Applied Biosystems. Serial dilutions of plasmid carrying the hygromycin-resistance gene or GAPDH were used to generate standard curves. 
Molecular modeling. The structures of DQ $\beta 1 * 0201$ and *0301 and gp42 were predicted using I-TASSER (29). The correctness of the modeled structure was evaluated by a confidence score (C-score) (43). $\mathrm{C}$-scores are normally in the range of $[-5,2]$. A higher $\mathrm{C}$-score indicates more confidence in the model, with a cutoff $\mathrm{C}$-score greater than -1.5 indicating models with a very high likelihood of correct topology (43). C-scores of the 5 best models of DQ $\beta 1 * 0201$ ranged from $[-3.22,-1.05]$, with a score of -1.05 for the top model. All 5 models had similar folds, including in the interface regions, and differed only in the unstructured region in the C-terminus between residues 186 and 261. C-scores of the 5 best models of DQ $\beta 1 * 0301$ ranged from [ $-3.35,-1.09]$, with a score of -1.09 for the top model. All 5 models of DQ $\beta 1 * 0301$ had similar folds as well, including in the interface regions, and differed only in the unstructured regions in the N-terminus between residues 1 and 35 and in the C-terminus between residues 223 and 261. The top model for gp42 had a C-score of 1.18 , while the other models had a score of -5 . Only the top predicted models were used for further structural analysis.

Statistics. All statistical analysis was done in R (44). To compare EBV-seropositive with EBV-seronegative subjects by HLA type, we used Fisher's exact tests to compare individual HLA-DQ $\beta 1$ types with all other HLA-DQ $\beta 1$ types for the odds of being EBV seronegative. The odds ratio of being EBV seronegative was defined as being greater than 1 , while the odds ratio of being seropositive was defined as less than 1 . Individual HLA-DQ $\beta 1 P$ values were adjusted to reduce the false discovery rate (45). Because experiments run on the same day were likely correlated, Wilcoxon signed-rank tests (46) were used to compare the binding activity of gp42 in cell lines that differed by HLA-DQ $\beta 1$. The false discovery rate was controlled by $P$-value adjustment within each independent experiment. Comparison between type 1 and 2 in the presence of F-2-1 over 6 different experiments on different days was performed using a linear mixed-effects model including a random intercept of experiment and adjusting for HLA-DQ $\beta 1$ type with the R packages lme4 $\left.{ }^{*}\right)$ and lmerTest $\left(^{* *}\right)$. To compare EBV infection between the HLA-DQ $\beta 1$ types, the Wilcoxon signed-rank test (46) was used with pairing by experimental day over 6 independent experiments. A $P$ value less than 0.05 was considered significant.

Study approval. The local Institutional Biosafety Committees approved blood donations at the NIH in Bethesda, Maryland, the Research Donor Program at the Frederick National Laboratory for Cancer Research in Frederick, Maryland, and the Blood Transfusion Center in Basel, Switzerland, and written consent was obtained from the volunteers before blood donations.

\section{Author contributions}

QL, WB, FA, WH, YH, and RLH performed the research. EG did statistical analysis. HM provided critical reagents and advice. $\mathrm{CH}$ provided clinical specimens. AR performed molecular modeling. QL and JIC designed the experiments, analyzed the data, and wrote the paper.

\section{Acknowledgments}

We thank Melanie Spriggs for plasmid pDC409-BZLF2 and Sharon Adams for assistance with HLA typing. We thank Toshio Kitamura for providing pMXs plasmids and Henri-Jacques Delecluse and Bill Sugden for the cell line expressing 293-EBV. This work was supported by the intramural research program of the National Institute of Allergy and Infectious Diseases. This project has been funded in whole or in part with federal funds from the National Cancer Institute, NIH, under contract HHSN261200800001E. The content of this publication does not necessarily reflect the views or policies of the Department of Health and Human Services, nor does mention of trade names, commercial products, or organizations imply endorsement by the U.S. Government.

Address correspondence to: Jeffrey I. Cohen, Building 50, Room 6134, 50 South Drive, MSC8007, NIH, Bethesda, Maryland 20892-8007, USA. Phone: 301.496.5265; E-mail: jcohen@niaid.nih.gov.

1. Balfour $\mathrm{HH}$, et al. Behavioral, virologic, and immunologic factors associated with acquisition and severity of primary EpsteinBarr virus infection in university students. J Infect Dis. 2013;207(1):80-88.

2. Faulkner GC, Burrows SR, Khanna R, Moss DJ, Bird AG, Crawford DH. X-Linked agammaglobulinemia patients are not infected with Epstein-Barr virus: implications for the biology of the virus. J Virol. 1999;73(2):1555-1564.

3. Longnecker R, Kieff E, Cohen JI. Epstein-Barr Virus. In: Knipe DM, et al., eds. Fields Virology. Philadelphia, PA: Lippincott Williams and Wilkins; 2013:1898-1959. 
4. Nemerow GR, Mold C, Schwend VK, Tollefson V, Cooper NR. Identification of gp350 as the viral glycoprotein mediating attachment of Epstein-Barr virus (EBV) to the EBV/C3d receptor of B cells: sequence homology of gp350 and C3 complement fragment C3d. J Virol. 1987;61(5):1416-1420

5. Ogembo JG, et al. Human complement receptor type 1/CD35 is an Epstein-Barr Virus receptor. Cell Rep. 2013;3(2):371-385.

6. Tanner J, Weis J, Fearon D, Whang Y, Kieff E. Epstein-Barr virus gp350/220 binding to the B lymphocyte C3d receptor mediates adsorption, capping, and endocytosis. Cell. 1987;50(2):203-213.

7. Li Q, et al. Epstein-Barr virus uses HLA class II as a cofactor for infection of B lymphocytes. J Virol. 1997;71(6):4657-4662.

8. Li Q, Turk SM, Hutt-Fletcher LM. The Epstein-Barr virus (EBV) BZLF2 gene product associates with the gH and gL homologs of EBV and carries an epitope critical to infection of B cells but not of epithelial cells. $J$ Virol. 1995;69(7):3987-3994.

9. Spriggs MK, et al. The extracellular domain of the Epstein-Barr virus BZLF2 protein binds the HLA-DR beta chain and inhibits antigen presentation. J Virol. 1996;70(8):5557-5563.

10. Sathiyamoorthy K, et al. Assembly and architecture of the EBV B cell entry triggering complex. PLoS Pathog. 2014;10(8):e1004309.

11. Stern LJ, et al. Crystal structure of the human class II MHC protein HLA-DR1 complexed with an influenza virus peptide. Nature. 1994;368(6468):215-221.

12. Haan KM, Kwok WW, Longnecker R, Speck P. Epstein-Barr virus entry utilizing HLA-DP or HLA-DQ as a coreceptor. $J$ Virol. 2000;74(5):2451-2454.

13. Mullen MM, Haan KM, Longnecker R, Jardetzky TS. Structure of the Epstein-Barr virus gp42 protein bound to the MHC class II receptor HLA-DR1. Mol Cell. 2002;9(2):375-385.

14. Ressing ME, et al. Epstein-Barr virus gp42 is posttranslationally modified to produce soluble gp42 that mediates HLA class II immune evasion. J Virol. 2005;79(2):841-852.

15. Ressing ME, et al. Interference with $\mathrm{T}$ cell receptor-HLA-DR interactions by Epstein-Barr virus gp42 results in reduced $\mathrm{T}$ helper cell recognition. Proc Natl Acad Sci USA. 2003;100(20):11583-11588.

16. Trowsdale J. The MHC, disease and selection. Immunol Lett. 2011;137(1-2):1-8.

17. Haan KM, Longnecker R. Coreceptor restriction within the HLA-DQ locus for Epstein-Barr virus infection. Proc Natl Acad Sci USA. 2000;97(16):9252-9257.

18. Balfour HH, Sifakis F, Sliman JA, Knight JA, Schmeling DO, Thomas W. Age-specific prevalence of Epstein-Barr virus infection among individuals aged 6-19 years in the United States and factors affecting its acquisition. J Infect Dis. 2013;208(8):1286-1293.

19. Condon LM, et al. Age-specific prevalence of Epstein-Barr virus infection among Minnesota children: effects of race/ethnicity and family environment. Clin Infect Dis. 2014;59(4):501-508.

20. de-Thé G. Epstein-Barr virus behavior in different populations and implications for control of Epstein-Barr virus-associated tumors. Cancer Res. 1976;36(2 pt 2):692-695.

21. Henle G, et al. Antibodies to Epstein-Barr virus in Burkitt's lymphoma and control groups. J Natl Cancer Inst. 1969;43(5):1147-1157.

22. Holling TM, Schooten E, Langerak AW, van den Elsen PJ. Regulation of MHC class II expression in human T-cell malignancies. Blood. 2004;103(4):1438-1444.

23. McShane MP, Mullen MM, Haan KM, Jardetzky TS, Longnecker R. Mutational analysis of the HLA class II interaction with Epstein-Barr virus glycoprotein 42. J Virol. 2003;77(13):7655-7662.

24. Miyadera H, Ohashi J, Lernmark Å, Kitamura T, Tokunaga K. Cell-surface MHC density profiling reveals instability of autoimmunity-associated HLA. J Clin Invest. 2015;125(1):275-291.

25. Ettinger RA, Liu AW, Nepom GT, Kwok WW. Exceptional stability of the HLA-DQA1*0102/DQB1*0602 alpha beta protein dimer, the class II MHC molecule associated with protection from insulin-dependent diabetes mellitus. J Immunol. 1998;161(11):6439-6445.

26. Greenbaum J, Sidney J, Chung J, Brander C, Peters B, Sette A. Functional classification of class II human leukocyte antigen (HLA) molecules reveals seven different supertypes and a surprising degree of repertoire sharing across supertypes. Immunogenetics. 2011;63(6):325-335.

27. Delecluse HJ, Hilsendegen T, Pich D, Zeidler R, Hammerschmidt W. Propagation and recovery of intact, infectious EpsteinBarr virus from prokaryotic to human cells. Proc Natl Acad Sci USA. 1998;95(14):8245-8250.

28. Erlich H, Lee JS, Petersen JW, Bugawan T, DeMars R. Molecular analysis of HLA class I and class II antigen loss mutants reveals a homozygous deletion of the DR, DQ, and part of the DP region: implications for class II gene order. Hum Immunol. 1986;16(2):205-219.

29. Yang J, Yan R, Roy A, Xu D, Poisson J, Zhang Y. The I-TASSER Suite: protein structure and function prediction. Nat Methods. 2015;12(1):7-8.

30. Shaw PL, Kirschner AN, Jardetzky TS, Longnecker R. Characteristics of Epstein-Barr virus envelope protein gp42. Virus Genes. 2010;40(3):307-319

31. Calderwood MA, et al. Epstein-Barr virus and virus human protein interaction maps. Proc Natl Acad Sci USA. 2007;104(18):7606-7611.

32. Gewurz BE, et al. Genome-wide siRNA screen for mediators of NF-kB activation. Proc Natl Acad Sci USA. 2012;109(7):2467-2472

33. Hutt-Fletcher LM. Epstein-Barr virus entry. J Virol. 2007;81(15):7825-7832.

34. Jabs WJ, Paulsen M, Wagner HJ, Kirchner H, Klüter H. Analysis of Epstein-Barr virus (EBV) receptor CD21 on peripheral B lymphocytes of long-term EBV- adults. Clin Exp Immunol. 1999;116(3):468-473.

35. Hui KM, Bidwell JL, eds. Handbook of HLA Typing Techniques. Boca Raton, FL: CRC Press; 1993.

36. Höcker B, et al. Epidemiology and morbidity of Epstein-Barr virus infection in pediatric renal transplant recipients: a multicenter, prospective study. Clin Infect Dis. 2013;56(1):84-92.

37. Durovic B, et al. Epstein-Barr virus negativity among individuals older than 60 years is associated with HLA-C and HLA-Bw4 variants and tonsillectomy. J Virol. 2013;87(11):6526-6529.

38. Edmond E, Steel CM, Ennis M, Cameron F, Dick H. HLA antigens in adults negative for antibody to Epstein-Barr virus (EBV). Tissue Antigens. 1981;18(4):252-257. 
39. Boyer KM, Sumaya CV, Cherry JD, Spencer MJ, Mickey MR, Terasaki PI. Histocompatibility antigens and humoral immunity to Epstein-Barr virus. Tissue Antigens. 1980;15(2):105-111.

40. Robinson J, Halliwell JA, Hayhurst JD, Flicek P, Parham P, Marsh SG. The IPD and IMGT/HLA database: allele variant databases. Nucleic Acids Res. 2015;43(Database issue):D423-D431.

41. Kanekiyo M, et al. Rational design of an Epstein-Barr virus vaccine targeting the receptor-binding site. Cell. 2015;162(5):1090-1100.

42. Kirschner AN, Omerovic J, Popov B, Longnecker R, Jardetzky TS. Soluble Epstein-Barr virus glycoproteins gH, gL, and gp42 form a 1:1:1 stable complex that acts like soluble gp42 in B-cell fusion but not in epithelial cell fusion. J Virol. 2006;80(19):9444-9454.

43. Zhang Y. I-TASSER server for protein 3D structure prediction. BMC Bioinformatics. 2008;9:40.

44. R Core Team. R: A language and environment for statistical computing. R Foundation for Statistical Computing, Vienna, Austria; 2015. https://www.r-project.org/. Accessed January 19, 2017.

45. Benjamini Y, Hochberg Y. Controlling the false discovery rate: a practical and powerful approach to multiple testing. $J R$ Stat Soc Series B Stat Methodol. 1995;57(1):289-300.

46. Hollander MW, Douglas A. Nonparametric Statistical Inference. New York, NY: Wiley; 1973. 